K. Brugmann, Der sogen. Akkusativ der Beziehung im Arischen usw. 121

\title{
Der sogenannte Akkusativ der Beziehung im Arischen, Griechischen, Lateinischen, Germanischen.
}

1.

Unter den syntaktischen Verwendungen der Akkusativform hat der wissenschaftlichen Deutung keine von jeher so viel Unbequemlichkeit und Schwierigkeit bereitet als die aus der Grammatik des Griechischen und des Italischen bekannte Gebrauchsweise, die man Akkusativ der Beziehung oder relationis, auch determinationis, in der lateinischen Grammatik meistens Accusativus Graecus nennt. In der Begrenzung, in der man diese Namen gewöhnlich anwendet, hat sie - von einer unten zu besprechenden Einzelheit abgesehen - in den neueren idg. Sprachen kein unmittelbares Analogon: z. B. für agriech. ảrŵ

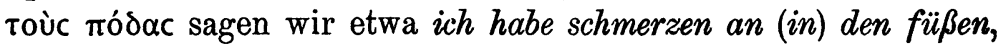

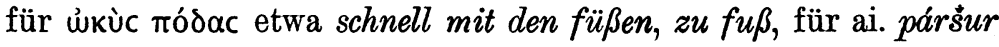
náma mānaví etwa die Manutochter, Paršu mit namen oder namens Paršu. Der moderne Indogermane kann daher hier nur schwer die besondere Art des Kasus nachempfinden.

Es ergibt sich naturgemäß vor allem die Frage, wie der 'Akkusativ der Beziehung' mit den sonstigen, auch in den neueren Sprachen geläufigen Verwendungen der Akkusativform innerlich zusammenhängt.

In den älteren Grammatiken begegnen allerlei philosophische Betrachtungen über unsern Kasusgebrauch, mit denen wenig gewonnen ist. So heißt es z. B. bei Kühner Ausführl. Gramm. d. griech. Spr. ${ }^{3}$ 2, 1, 303 (vgl. S. 315 ff.), Ausdrücke wie $\alpha \lambda r \omega ̂$ тทेv

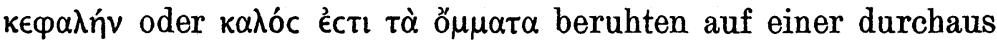
einfachen und kindlichen Anschauung, während der präpositionale Ausdruck der modernen Sprachen sich auf eine verstandesmäßige Auffassung des in Wirklichkeit bestehenden Verhältnisses der Dinge gründe.

Nebelig und nichts fördernd wie solche Anschauung und Behandlung der Sache ist auch ihre am weitesten verbreitete Benennung als Akkusativ der Beziehung oder des Bezugs. Gleichwie so viele Termini der Grammatik, entstammt sie nicht einem unbefangenen und umsichtigen Eindringen in die Natur der Erscheinung und ihre Eigenart, sondern jenem schon in der vorwissenschaftlichen Zeit der Grammatik bei der Registrierung 
und Einteilung der verschiedenen Verwendungen einer Sprachform naturgemäß sich geltend machenden Bezeichnungsbedürfnis, das in der Regel mit dem ersten besten, was sich darbot, zufrieden gewesen ist. Man wählte die Bezeichnung 'der Beziehung',

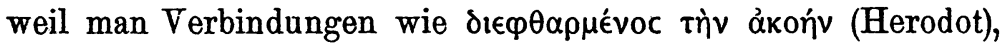

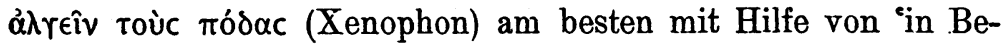
ziehung auf, in bezug auf' glaubte sich zugänglich machen und verdeutlichen zu können. Dies mag angehen. Aber die Übersetzung mit diesen Ausdrücken paßt schon z. B. bei åvì

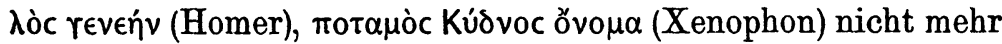

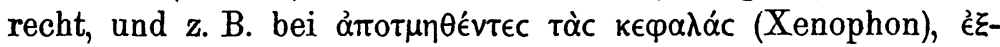

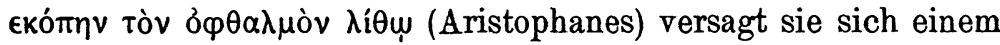
natürlichen Empfinden vollends. Trotzdem hat der Name sich eingebürgert, und wie das so zu gehen pflegt, an seiner Hand wurde der Begriff der Beziehung auf etwas für die 'Grundbedeutung' der ganzen Gruppe von einschlägigen Anwendungen unseres Kasus ausgegeben.

Da dieser Akkusativ von jeher eine Crux auch des Schulunterrichts gewesen ist, so erlaube ich mir eine meiner klarsten Erinnerungen aus meiner Schulzeit zur Sprache zu bringen. Als ich in Tertia saß, wurden wir vom Lehrer streng angehalten, die Xenophonstelle, die ich soeben berührt habe, An. 2, 6, 1 oi

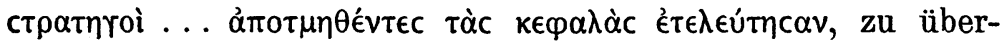
setzen mit 'die Feldherrn starben, abgeschnitten (indem sie abgeschnitten wurden) in bezug auf ihre Köpfe', auf daß wir das richtige grammatische Verständnis des Satzes gewännen und den Geist dieses Akkusativs genau erfaßten. Kein Wunder, daß infolge dieses und gleichartiger Verdeutlichungsexperimente mir und wohl auch manchem meiner Mitschüler der Akkusativ der Beziehung auf lange Zeit den Eindruck einer höchst wundersamen und verschrobenen Redeweise gemacht hat; die Verschrobenheit legten wir natürlich den alten Griechen selbst zur Last. Daß der technische Name hier, wie in so vielen Fällen, höchstens als ein Notbehelf berechtigt sei, und daß jene Wiedergabe mit 'in bezug auf' an sich ebensoviel und ebensowenig Berechtigung habe als wenn jemand etwa sperat victoriam mit 'er hofft in bezug auf Sieg' oder Romam proficiscitur mit 'er bricht auf in bezug auf Rom' verdeutlichen wollte, sagte uns niemand. Ich weiß nun nichts Näheres darüber, auf welche Weise sich heute unsere Gymnasiallehrer im Unterricht mit dem 'Akkusativ der 
Beziehung' abfinden. Da aber die Benennung nicht aufgegeben ist und die liebe Gewohnheit, bei mangelndem Verständnis einer sprachlichen Erscheinung sich an den Namen zu halten, mit dem sie getauft ist, ebenfalls noch keineswegs ausgestorben ist, so vermute ich, daß auch heute noch die Verdeutschung mittels in bezug auf ihre Rolle in der Schulpraxis nicht ganz ausgespielt hat. Zum Teil wird man, wo man mit diesem Akkusativ nichts Rechtes anzufangen weiß, wohl auch noch andere Praktiken anwenden.

Einen Versuch, nicht nur vom Standpunkt der sprachgeschichtlichen Forschung aus, sondern zugleich und vornehmlich pädagogisch dem 'Akkusativus der Beziehung' beizukommen, finde ich bei P. Cauer in seiner Grammatica militans $285 \mathrm{ff}$. Was dieser Gelehrte in ersterer Hinsicht bietet, befriedigt nicht. Um so annehmbarer ist es dagegen, wenn er, nach dem Vorgang von Ziemer, den Schüler auf den Akkusativ in deutschen partizipialen Wendungen wie den kopf unbedeckt, die hände auf den rücken gelegt hinweist. Hierauf wird unten (§ 7) zurückzukommen sein.

2.

Über den in Rede stehenden Kasusgebrauch kann, das ist heutzutage selbstverständlich, nur dadurch Klarheit kommen, daß es gelingt, ihn entwicklungsgeschichtlich richtig einzuordnen. Damit ist die Aufgabe bezeichnet, die uns im folgenden zu beschäftigen hat.

Gut bemerkt Paul Prinzipien ${ }^{4}$ 153: "Der Akkusativ bezeichnet überhaupt jede Art von Beziehung eines Substantivums zu einem Verbum, die sich außer der des Subjekts zu seinem Prädikate denken läßt. Dennoch aber können wir ihn nicht in jedem einzelnen Falle, in den eine solche allgemeine Beziehung stattfindet, anwenden, und schon in der indogermanischen Grundsprache war das unstatthaft, wenn auch die Verwendung noch eine viel freiere und ausgedehntere war... Die Angabe einer einzigen, alles umfassenden Bedeutung genügt daher nicht; wir müssen verschiedene allmählich selbständig gewordene Verwendungsweisen neben einander stellen. Hier kommt nun aber hinzu, daß auch in der Beziehung auf einzelne Verba ein fester Usus in bezug auf Gebrauch oder Nichtgebrauch des Akkusativs und eine Spezialisierung der Bedeutung eingetreten ist". 
Hiernach fragt es sich zunächst: ist die Gebrauchsweise, die man Akkusativ der Beziehung nennt, und deren Entstehung und Ausbildung in vorhistorische Zeiten der Sprachgeschichte fällt ${ }^{1}$ ), ebenso alt wie diejenigen Anwendungen des Kasus, die daneben in den idg. Sprachen auftreten und die schon in der Zeit der idg. Urgemeinschaft feste - wenn auch später im einzelnen teils weiter ausgebreitete, teils eingeschränkte - Gebrauchstypen gewesen sind? Oder ist sie erst aus diesen abgeleitet? Im ersteren Fall wäre man mit der wissenschaftlichen Erklärung bald fertig; man käme über die Feststellung der Tatsache kaum hinaus, daß sich im 'Akkusativ der Beziehung' eine uralte Gebrauchsweise der der Subjektsform des Substantivs gegenüberstehenden Objektsform erhalten habe (vgl. F. Hanssen Philosophemata zur lat. Syntax, Comment. in hon. Guilelmi Studemund S. 112f.). Im anderen Falle dagegen erscheint es im Hinblick auf die uns zu Gebote stehenden Erkenntnismittel als eine nicht hoffnungslose Aufgabe, den Punkt oder die Punkte innerhalb der Gesamtsphäre des Akkusativgebrauchs ausfindig zu machen, von wo unsere Anwendung ausgegangen ist.

Nun ist von vorn herein die Wahrscheinlichkeit durchaus dafür, daß wir es mit einer solchen sekundären Entwicklung zu tun haben. Dafür spricht besonders der Umstand, daß in der Mehrzahl der Fälle der Akkusativ nicht von einem Verbum, sondern von einem Nomen abhängt; denn der ursprünglichere Gebrauch des Akkusativs muß ja der adverbale gewesen sein. So hat man denn auch in neuerer Zeit den 'Akkusativ der Beziehung” stets, so viel ich weiß, als eine Fortsetzung der einen oder der anderen von den sonstigen überlieferten Akkusativverwendungen betrachtet. Teils den ganzen in Rede stehenden Gebrauch in den verschiedenen Sprachen, teils diese oder jene einzelne Gruppe innerhalb desselben findet man so bereits erklärt von Delbrück Die Grundlagen der griech. Synt. $32 \mathrm{ff}$, Grundriß 3, 387 ff., mir Griech. Gramm. ${ }^{3} 381$ f., Hanssen a. a. O., Cauer a. a. O., Neckel IF. 19, 253, Gaedicke Der Acc. im Veda 216 ff., Gray IF. 11, 307 ff., Foy IF. 12, 172 ff. Auch ältere klassische Philologen haben sich schon auf diesen Standpunkt gestellt, z. B. G. Hermann Vig. Idiot. Gr. 882.

1) Hierbei ist vom 'Accusativus Graecus' des Lateinischen keineswegs ganz abzusehen. S. § 4 S. $132 \mathrm{ff}$. 
Ich gebe nunmehr meine Auffassung der Entstehungsgeschichte. Im Verlauf der Darstellung wird sich Gelegenheit bieten, zu dieser und jener von den bisher vorgetragenen Auffassungen Stellung zu nehmen; sie alle vorzuführen und zu kritisieren scheint mir überflüssig.

3.

In allen Fällen, wo ein 'Akkusativ der Beziehung' deutlich vorliegt, war die Sprache ausgegangen von solchen Ausdrucksweisen, in denen das betreffende Substantiv einstens als Subjekt eines einfachen Satzes vorgestellt war. Dieser Satz war Glied einer umfassenderen Gesamtvorstellung und anderm innerhalb derselben psychologisch untergeordnet. Die Wahl einer andern Kasusform statt des Nominativs wurde dadurch bestimmt, daß grammatisch-formale Einverleibung in den übergeordneten Satz für das betreffende Substantiv die Nominativform ausschloB. Daß man aber gerade zum Akkusativ griff, geschah nach dem Vorbild von Objektsakkusativen, die, neben einem andern zum selben Verbum gehörigen Objektsakkusativ stehend, diesem untergeordnet, ihm gegenüber degradiert waren.

Dies das Wesentliche. Es bedarf näherer Ausführung, und ich fasse zunächst solche Konstruktionen für sich ins Auge, im Anschluß an die - zunächst theoretisch betrachtet - 'Akkusative der Beziehung’ sich überhaupt haben entwickeln können.

Hat ein Verbum einen Akkusativ des äußern oder des innern Objekts bei sich, so tritt für ihn bei passivischer Ausdrucksweise der Nominativ ein, einerseits z. B. domum aedifico - domus aedificatur, und so in allen indogermanischen Sprachen, anderseits z. B. pugnam pugno - pugna pugnatur, und ebenso

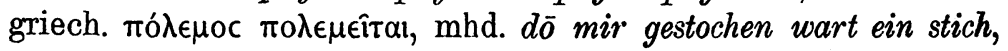
ai. viṣ̂nukkramáh kramyantē 'Viş̌nuuschritte werden geschritten'. Hängen aber zwei Akkusative von einem Verbum ab, die nicht lediglich im kopulativen Verhältnis zu einander stehen, so erscheinen beim Passivum für die beiden Akkusative nur dann zwei Nominative, wenn schon beim Aktivum die beiden Akkusative in einem Verhältnis zu einander waren, das dem Verhältnis von Subjekt und Prädikat entspricht, z. B. Ciceronem consulem fecerunt - Cicero consul factus est. Besteht dieses Verhältnis nicht zwischen beiden zum Verbum activum gehörigen Akkusativen, so kann es ein solches sein, daß man kurz den 
einen Akkusativ als Hauptobjekt, den andern als Nebenobjekt bezeichnen kann. Da kommt denn das, was dem Sprechenden das Hauptobjekt ist, bei passivischer Wendung in den Nominativ, das andere hingegen bleibt in der Akkusativform. Dieses Verbleiben des einen Objekts im Akkusativ ist hier schon in der aktivischen Ausdrucksweise durch die Art der Beziehung, in die die beiden Akkusative zu einander gesetzt waren, ebenso vorbereitet worden, wie der doppelte Nominativ bei Cicero consul factus est durch die Art des Verbältnisses der Akkusative in der aktivischen Redeweise vorbereitet war.

Für uns kommen hier nun drei Klassen von Verbindungen zweier Akkusative in Betracht: erstens die Verbindung eines Akkusativs der Person und eines Akkusativs der Sache, wie

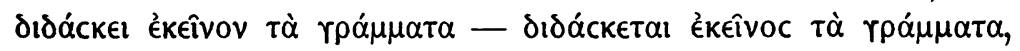
zweitens die Verbindung eines Akkusativs, der ein Ganzes, und eines Akkusativs, der den speziell von der Tätigkeit be-

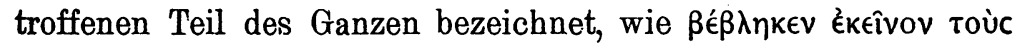

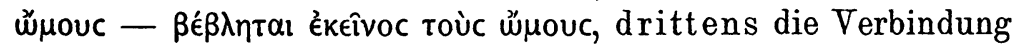
eines Akkusativs des äußeren und eines Akkusativs des inneren

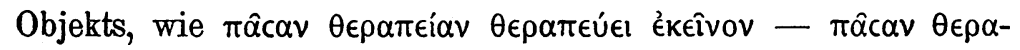

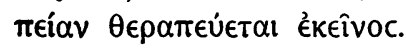

Bei den zwei ersten Klassen von Verba sind beide Akkusative zugleich von Haus aus äußeres Objekt gewesen, und sie sind es, die nach meinem Dafürhalten die Vorbilder für das geliefert haben, was den 'Akkusativ der Beziehung' ausmacht. Die dritte Klasse darf man nur dann als beteiligt bei der Entwicklung dieses Akkusativgebrauchs ansehen, wenn man - wie zuweilen geschehen ist - dem Terminus Akkusativ der Beziehung einen Gebrauch zugesteht, der über das hinausgeht, wofür er anfangs angewendet worden ist, einen Gebrauch, der nicht $\mathrm{zu}$ empfehlen ist (vgl. § 10).

1. Zunächst also die Verba des Lehrens, Beraubens, Hehlens u. a., bei denen, in derselben Weise in verschiedenen idg. Sprachen zugleich, eine Person (beziehungsweise eine Sache, die als Person gedacht ist) und eine Sache, die von der Tätigkeit betroffen werden, im Akkusativ erscheinen, z. B. ich lehre dich diese sprache, lat. te litteras doceo. Diese Konstruktionsweise hat als aus uridg. Zeit stammend zu gelten. Siehe Delbrück Grundr. 3, 383ff. Man hat es hier mit einer sogenannten Konstruktion åmò koเvov̂ zu tun, insofern als das Verbum den zwei Objekten gegenüber einen 
verschiedenen Sinn hat (vgl. Gaedicke Der Acc. im Veda 265). Dabei kann die Beziehung, in der die beiden Objekte zu einander sich befinden, eine zwiefache sein:

Entweder steht die Person gegenüber der Sache im Vordergrund: dann tritt z. B. bei dem Satz ich lehre ihn diese sprache die Vorstellung ins Bewußtsein 'er lernt diese Sprache', und bei passivischer Ausdrucksweise formt sich die Person zum Satzsubjekt, erscheint also in der Nominativform. Das ist der gewöhnlichere Fall. So mhd. daz lantliut wart gelèret den gelouben, nhd. (Goethe) das schlimmste, was uns widerfährt, das

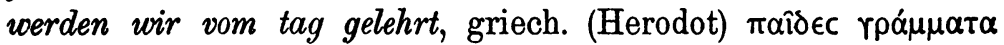

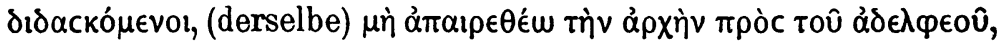

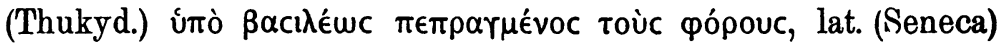
docebatur blanditias, (Plaut.) ut moneatur officium suom, (Cic.) rogatus sum sententiam, (Ovid) poscor meum Laelapa, (Nepos) id Alcibiades diutius celari non potuit, ai. RV. 9, 74, 4 ätmanván nábhō duhyate ghrtám páyah 'belebter Wolke wird entzogen fette Milch' (vgl. den doppelten Akkusativ 9, 107, 5 duhāná údhar divyám mádhu priyám 'aus des Himmels Euter lieben Meth herausmelkend'), klass. bānēna yuddhayoggyam arị harō yācitah 'von Bāna wurde Çiva um einen dem Kampf gewachsenen Feind gebeten' ").

Oder die Vorstellung der Sache hat das Übergewicht, so daß z. B. mit has res me celant sich die Vorstellung 'hae res me fugiunt' verbindet. Dann tritt beim Passiv die Sache in der Nominativform auf, wie (Plaut.) quor haec celata me sunt?, mhd. das golt ist mich harte lange übele verholn, ahd. wanta ist firholan iuwih al, wanne druhtin queman scal, mhd. moeren, die mich verswiget waren, mhd. sage uns, wie der name dich gelèret si.

Daß die Umsetzung des einen von den beiden Kasus, die sich mit dem aktivischen Verbum verbanden, in den Nominativ nicht gerade davon abhängig war, daß er ein Akkusativ war, zeigen die Fälle, wo bei der Umwandlung ins Passiv ein Dativ der Person neben einem Akkusativ der Sache Satzsubjekt wurde,

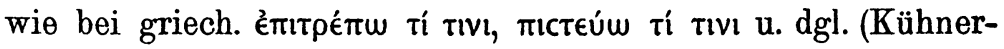

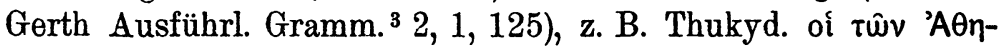

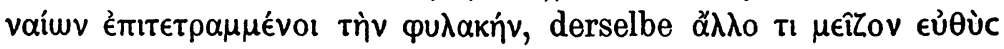

1) Für das Indische vgl. Gaedicke Der Acc. im Veda 267, Speyer Sanskrit Syntax S. 35. 37, Ved. u. Sanskrit-Synt. S. 8. - Lit. esù mokinams tạ rãsztg ('ich werde das Schreiben gelehrt') u. dgl. mag Germanismus sein. 


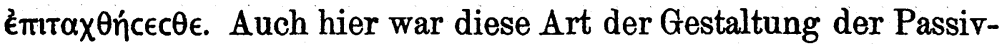
konstruktion geleitet durch die Konstruktion sinnverwandter

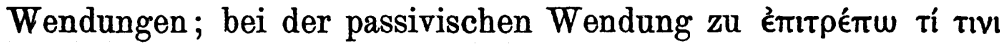
z. B. schwebte vor, daß ein er etwas zugewiesen erhält oder dgl. ${ }^{1}$ ).

Nur in bezug auf den Akkusativ der Sache hat sich nun mit der Zeit das Sprachgefühl derart geändert, daß sich die Vorstellung des Objektsverhältnisses verflüchtigte und dem Zusammenhang nach ein 'Akkusativ der Beziehung' daraus wurde.

2. Die Verba, bei denen von den beiden ron ihnen abhängenden Objektsakkusativen der eine ein Ganzes, in der Regel eine Person als Ganzes, der andere einen speziell von der Tätigkeit betroffenen Teil bezeichnet. Häufig erscheint dieser zwiefache Ak-

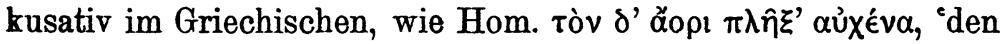
traf er mit dem Schwert, den (seinen) Hals’ = 'den traf er am Hals', seltener im Indischen, wie AV.átrāinān indra vrtrahann ugrố mármāni vidhya 'dann, o Indra, Vrtratöter, triff sie als Held, die (ihre) Bößen' = 'triff sie auf ihre Blößen'. Vgl. Delbrück Grundriß 3,385f.

Aus dem Awesta ist ein sicherer Beleg nicht nachgewiesen. Vielleicht gehört hierher V. 18, 38 hō mąm ava 9 a varanąn nijainti ya $a$ usw. Scheftelowitz ZDMG. 57, $159 \mathrm{f}$. nennt vərəną einen 'Akkusativ der Beziehung' und übersetzt 'dieser schlägt mich so in bezug auf die Schwangerschaft, als ob ..., während Bartholomae Altiran. Wtb. 493 vermutet, mam vertrete den Genitiv, und dem zu var- 'schwanger machen, werden' gehörigen varəna- den konkreten Sinn 'Leibesfrucht' gibt. Zulässig scheint mir jedenfalls die Auffassung 'dieser schlägt (richtet zugrunde) mich, die (meine) Leibesfrucht ebenso, als ob ....

Auch bietet, wie Neckel IF. 19, 251 gesehen hat, der germanische Zweig möglicherweise indirekte Belege durch die aisl. Verba wie halshoggua 'den Hals abschlagen’, halsliósta 'an den Hals schlagen' u. dgl. Denn eine Konstruktion mit doppeltem Akkusativ kann Sätzen wie onnur kuez hafa halslostit hana 'eine andere sagte, sie habe sie an den Hals geschlagen' zugrunde gelegen haben.

1) Das Gleiche gilt für den passivischen Ausdruck natürlich in dem Fall, daß das Verbum keinen Akkusativ der Sache bei sich hat, z. B.

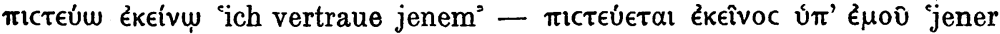
findet Vertrauen bei mir'. So erscheint auch für den vom aktiven Verbum

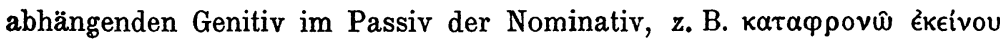

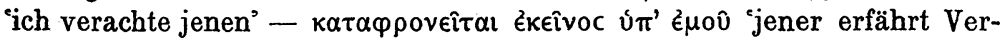
achtung von mir' (Kühner-Gerth a.a. 0. 124f.). 
Einige Beispiele, die in der lateinischen Literatur begegnen, gelten als Gräzismus (vgl. Landgraf Wölfflins Archiv 10, 215). Man sieht aber nicht ein, warum ein Satz wie Plaut. te Venus eradicet caput atque aetatem tuam oder wie Verg. Latagum saxo atque ingenti fragmine montis occupat os faciemque adversam (C. F. W. Müller Syntax des Nom. u. Akk. 131) nicht ebenso ein echt lateinisches Spracherzeugnis gewesen sein soll, wie etwa Otfrids druhtīn wasg mih al, houbit ioh thie fuazi "wasch mich gänzlich, Kopf und Füße' sicher echt deutsch gesagt war (Erdmann Untersuch. über die Synt. der Sprache Otfr. 2, 130).

Diese Art von doppeltem Akkusativ, die zu dem sogenannten

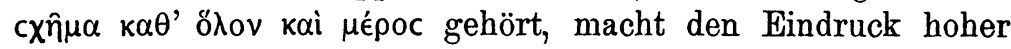
Altertümlichkeit, und man geht schwerlich fehl, wenn man auch ihr urindogermanisches Alter zuschreibt. Damit ist freilich nicht gesagt, daß alles, was in den historischen Zeiten zu ihr gerechnet werden kann, mechanische Nachahmung von älteren und ältesten Mustern gewesen sein müsse. Denn das zugrunde liegende Verhältnis des zweiten Akkusativs zum ersten war ein einfaches appositionelles, wie es in jeder Sprache immer vorgekommen ist,

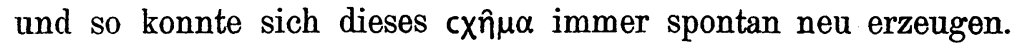

Daß der Teilbegriff tatsächlich nur eine einfache Apposition zum Hauptbegriff gebildet hat, daß die beiden Akkusative also von Haus aus derselben Akkusativklasse angehört haben, wird

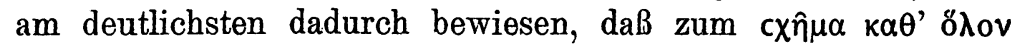

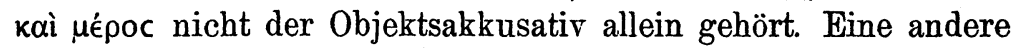
Gattung von Akkusativ erscheint RV. 1, 80, 15 nahi nú yád adhimmásíndra kố viryà paráh `denn, soviel wir wissen, geht niemand über Indra, (über) seine Kräfte'; hier hängen die Akkusative beide von der Präposition ab (Delbrück Grundriß 3, $385 \mathrm{FuBn}$. 1). Zweifachen Dativ dieser Art hat man z. B. $\wedge 11$ 'Axaııîcıv dè

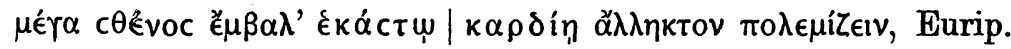

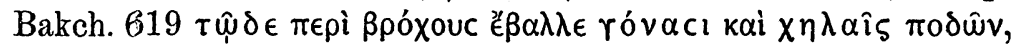

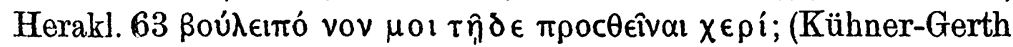
a. a. O.). Ferner scheint es mir natürlicher, den doppelten Genitiv

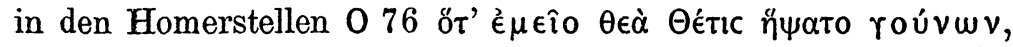

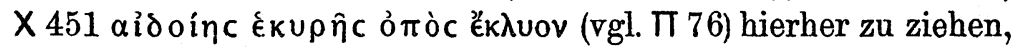
als daß man, wie die späteren Griechen nach Maßgabe ihres veränderten Sprachgefühls taten, den vorderen Genitiv als Possessivus dem folgenden Genitiv untergeordnet sein läßt. Schließlich darf auch an den doppelten Nominativ in Sätzen wie H 175 oi

Indogermanische Forschungen XXVII. 


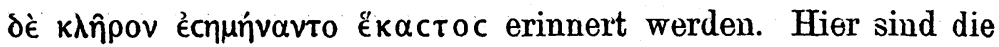
beiden Substantivbegriffe freilich anderer Art, nämlich der Begriff einer Menge und der eines Individuums, aber es waltet wenigstens das gleiche Appositionsverhältnis.

In unserm speziellen Fall nun, in den aktivischen Wen-

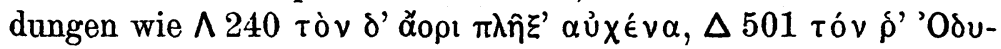

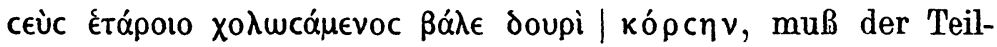
akkusativ seinen Wert als Apposition zum Akkusativ des Ganzen frühe eingebüßt haben. Das Verhältnis der psychologischen Unterordnung des Teilakkusativs unter den andern Akkusativ blieb bestehen, nur war es eben nicht mehr das appositionelle. Bewiesen wird das wieder durch die passivische Wendung, wie

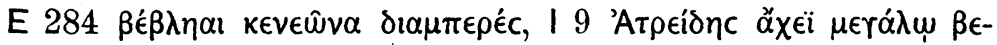

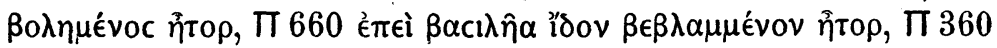

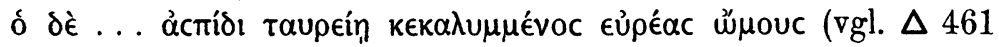

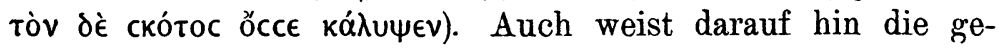
legentlich vorkommende, allerdings noch nicht für Homer sicher bezeugte Vorausstellung des Akkusativs des Teils ${ }^{1}$ ). Daß die Umwertung des Teilakkusativs unter dem Einfluß der Konstruktion anderer Verba mit dem Akkusativ vor sich ging, ist an sich wahrscheinlich. Doch ist hierüber schwer ins Reine zu kommen. Bei solchen Verba, die den Begriff der Bewegung eines Gegen-

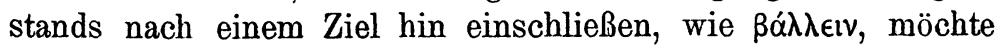
man eine gewisse Einwirkung des sogenannten Akkusativs des Ziels oder der Richtung (oủpavòv îke) annehmen ${ }^{2}$ ). Jedenfalls hat Delbrück Grundr. 3, 385 f. recht, wenn er sagt, der Akkusativ

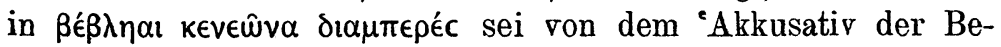
ziehung' nicht mehr zu unterscheiden.

4.

Weiter stimme ich Delbrück (S. 392) auch darin bei, daß

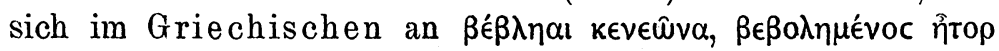
u. dgl. die Gewohnheit ausgebildet habe, mit passivischen Formen

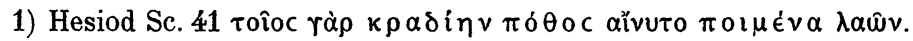

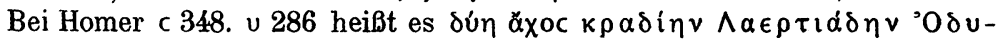

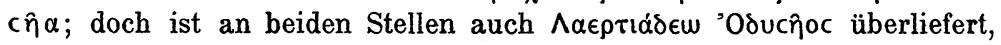
und zwar ist c 348 der Genitiv, $v 286$ der Akkusativ besser bezeugt.

2) Verba mit dem Akkusativ des Ziels zeigen zum Teil selbst den

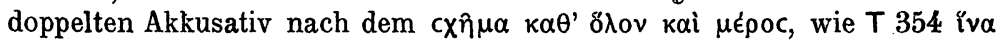

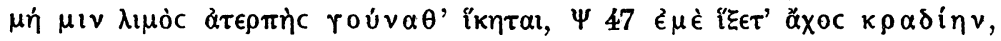

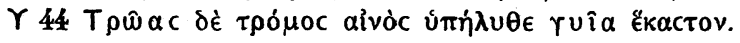


das von der Handlung betroffene Glied auch dann im Akkusativ zn verbinden, wenn das Verbum im Aktiv oder Medium nicht mit dem Akkusativ des Ganzen und des Teiles konstruiert wurde.

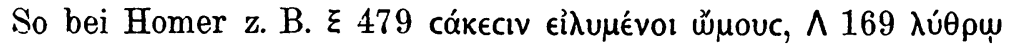

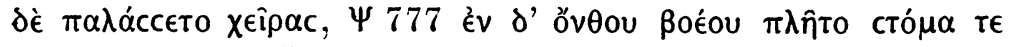

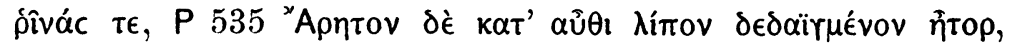

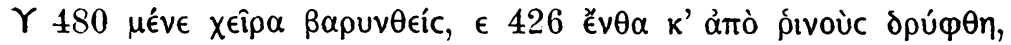

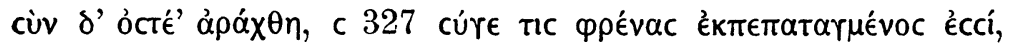

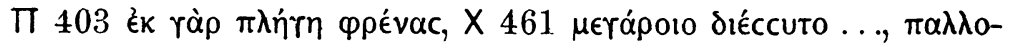

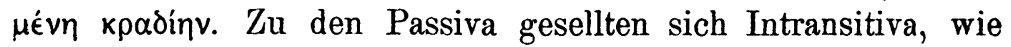

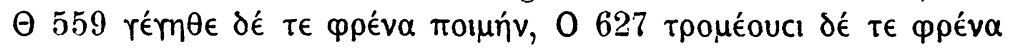

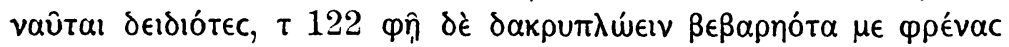
oivw. Diese Konstruktionsweisen blieben auch in der nachhome-

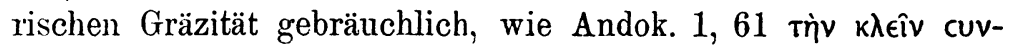

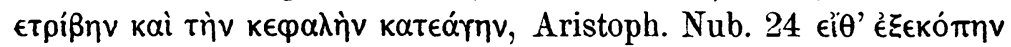

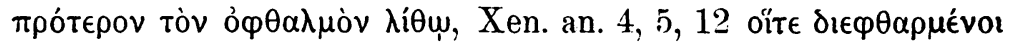

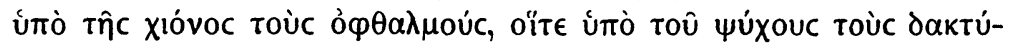

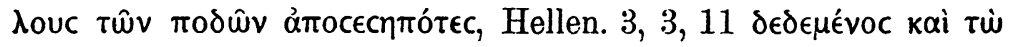

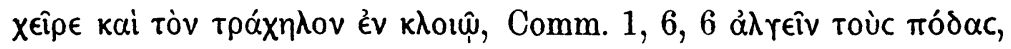

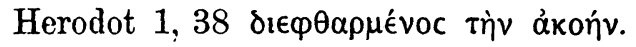

Durch Vermittlung der Partizipia hat sich im Griechischen diese Konstruktion mit dem Akkusativ weiter auf zahlreiche Adjektiva verpflanzt, welche Zustände und Eigenschaften von Personen angeben. Neben diesen Adjektiva erscheinen zum Teil aber auch wieder etymologisch zugehörige Verbalformen, Formen des Partizipiums oder des Verbum finitum. Zunächst Wörter, durch die Zustände oder Eigenschaften des Körpers

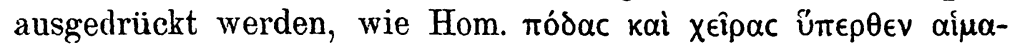

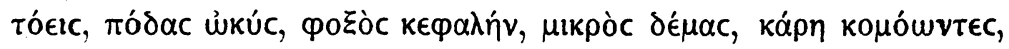

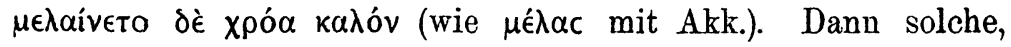
mit denen Zustände oder Eigenschaften des inneren Menschen

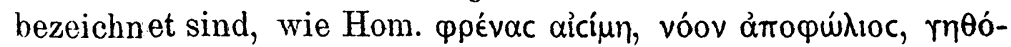
cuvoc kn̂ $\rho$, wozu die schon oben erwähnten Verba rérn $\theta \alpha$ and

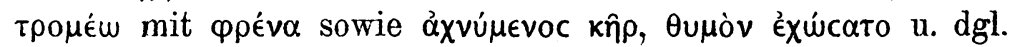
zu stellen sind. Ferner 'gleich', 'ähnlich' mit zugehörigen Verben,

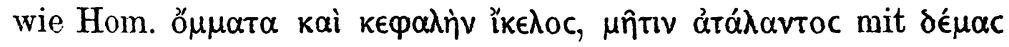

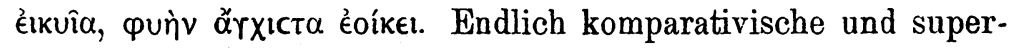

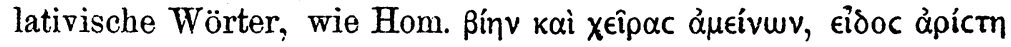

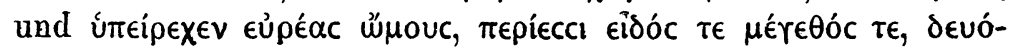
$\mu \in$ vov $\varphi \rho \in ́ v \alpha c$. Ebenso wiederum in der nachhomerischen Gräzität 


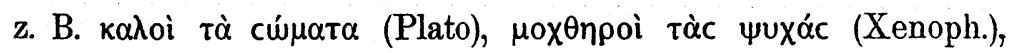

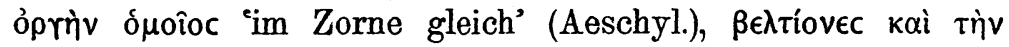

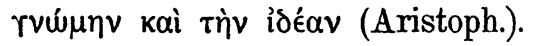

Allen diesen griechischen Wendungen stehen gleichartige im Lateinischen gegenüber; sie gelten bekanntlich als Gräzismus. S. zuletzt hierüber Landgraf Wölfflins Archiv 10, $209 \mathrm{ff}$. 376, C. F. W. Müller Syntax des Nom. und Akk. $111 \mathrm{ff.,} 127 \mathrm{ff}$.

Zunächst passivische Verba, ganz vorwiegend Participia Perfecti Pass., mit dem Akk., wie Lukr. mentes perculsa, Catull. contecta pectus amictu, restrictus membra catena, Verg. turbatus pectora, Sall. dedecores inultique terga caedebantur, Liv. femur ictus.

Dann Adjektiva mit dem Akkusativ, wie Verg. saucius pectus, Ov. nigra pedes, Hor. mitior animum, Sil. ferus mentem, Verg. os umerosque deo similis.

Zuweilen auch Intransitiva mit Akkusativ, wie Lukr. tremit artus.

Dieser ganze sogen. Accusativus Graecus der römischen Sprache ist nun nicht in dem Sinne Gräzismus, als ob damit etwas, was den syntaktischen Gewohnheiten der römischen Sprache völlig fremd gewesen wäre, aus dem Griechischen herübérgenommen worden sei. Vielmehr wurzelt er, wie schon öfters mit Recht gesagt worden ist, in echt lateinischem volkstümlichen Akkusativgebrauch. An diesen haben die Römer das, was sie bei den Griechen vorfanden, unwillkürlich unmittelbar anempfunden. Und so ist als Gräzismus im Grunde nur die starke Kultivierung dieses Akkusativgebrauchs von der Zeit an, wo die römische Sprache, namentlich die Sprache der Dichter, unter den Einfluß der griechischen Sprache kam, zu bezeichnen; wobei denn im einzelnen manches natürlich auch direkte Nachahmung, einfache Übersetzung griechischer Ausdrücke war (Nachweise bei Landgraf a. a. O.) ${ }^{1}$ ). Daß man dabei heute genau noch die Grenze aufweise, wo jedesmal der griechische Einfluß eingesetzt hat, ist nicht zu verlangen. Dazu müßten wir vom Sprechen der Römer und von den Römern als Sprechern unendlich viel mehr wissen, als wir aus der trümmerhaften Überlieferung entnehmen können.

1) Ganz ähnlich ist es mit den meisten syntaktischen Gräzismen im Lateinischen, aber auch den meisten syntaktischen Romanismen im Deutschen, den meisten syntaktischen Germanismen und Slavismen im Litauischen usw. usw. 
Echt lateinisch war zunächst der Akkusativ in den passivischen Wendungen wie litteras docetur, litteras doctus, aliment rogatur, sententiam interrogatus, illud admonitus, diem celatus (S. 127). Hieran schlossen sich analogisch passivische Ausdrücke an in solchen Fällen, wo das Verbum im Aktivum nicht den doppelten Akkusativ regierte ${ }^{1}$ ). Dies geschah aber im $\mathrm{Zu}-$ sammenhang damit, daß die betreffenden Verba in der deponentialpassivischen Form großenteils von alters her zugleich passivischen und medialen Sinn hatten. So induor vestem, indutus vestem, gleichwie umbr. perca arsmatiam anouihimu 'virgam ritualem induitor' (vgl. Verf. IF. 5, 112 f. 131, v. Planta Osk.-umbr. Gramm. 2, 410); es ist also unberechtigt, bei Stellen wie Plaut. Men. 192 non ego te indutum foras exire vidi pallam?, Ov. met. 7, 182 vestes induta recinctas, Verg. Aen. 7, 640 loricam induitur von Gräzismus zu sprechen (vgl. hierzu Landgraf $218 \mathrm{ff}$.). Für die Frage, wie weit Gräzismus oder echt italisch, ist ferner nicht unwichtig osk. T. B. 19 pis ceus Bantins fust censamuv esuf in. eituam poizad ligud iusc censtur censaum angetuzet 'qui civis Bantinus erit, censetor ipse ${ }^{2}$ ) et pecuniam, qua lege ei censores censere proposuerunt'. Denn ebenso heißt es bei Cic. pro Flacco 32,80 census es mancipia Amyntae; ... ac primo quidem pertimuit, cum te audisset servos suos esse censum (v. Planta. a. a. O., Buck Grammar 199). Hier kann von griechischem Einfluß nicht wohl die Rede sein.

Wenn es nun echt lateinisch vestem induo, induor, indutus hieß und echt lateinisch corpus induo, induor war, so kann auch corpus indutus echt lateinisch gewesen sein und weiter dann auch caput velati, contecta pectus, cinctus tempora, succincti corda u. dgl. So war also der Boden für den ausgedehnteren Gebrauch. den durch die augusteischen Dichter der 'Akkusativ der Beziehung' beim Participium Perf.Pass. und weiter bei den Adjektiva

1) Bei dem einen oder anderen Verbum, das hierher zu gehören scheint, mag jedoch diese Konstruktion des Aktivs in vorhistorischer Zeit existiert haben und unmittelbar hierauf die in Rede stehende Passivkonstruktion fußen. Von dem doppelten Akkusativ im Spätlatein, wie Vulg. Marc. 15, 17 induerunt eum purpuram (Landgraf S. 220, C. F. W. Müller S. 130 f.), ist hier natürlich abzusehen.

2) Für die Auffassung von eituam ist es gleichgültig, ob man esuf; das Adverbium ist und dem griech. aủ $\delta \theta_{\imath}$ entspricht (Verf. Demonstr. 125), als 'an Ort und Stelle, daselbst' oder als 'an sich selbst, in bezug auf sich selbst' erklärt. 
erfahren hat, bei den Römern selbst schon wohl vorbereitet, und als Gräzismus wird man vielleicht nur den Übergang vom Partizip mit Akkusativ zum Adjektiv mit Akkusativ zu bezeichnen haben. Wie klein war aber der Schritt z. B. von nudatus pedem zu nudus pedem, von vulneratus pectus $\mathrm{zu}$ saucius pectus!

Wie weit man im Lateinischen von einem 'Akkusativ der Beziehung' reden will, ist, ähnlich wie in Griechischen, im Grunde nur Sache der Verabredung. Wenn jemand so z. B. beide Akkusative in Ov.met. 7, 182 (Medea) nuda pedem, nudos umeris infusa capillos oder in Verg. Aen. 1,320 (Harpalyce) nuda genu nodoque sinus collecta fluentes bezeichnen will, so ist sicher nichts dagegen einzuwenden.

Diesen ganzen Gebrauch des Lateinischen aber als Accusativus Graecus zu bezeichnen, ist jedenfalls unzweckmäßig, weil der Name irre führt. Wenn man den Namen Beziehungsakkusativ in der Sprachwissenschaft überhaupt beibehalten will, sollte man ihn auch für die lateinische Sprache (wie für das Oskische und das Umbrische!) benutzen und hätte dann hier nur von seiner unter dem Einfluß der griechischen Sprache erfolgten größeren Ausdehnung zu sprechen.

\section{5.}

Der Gebrauch des 'Akkusativs der Beziehung' in den bis jetzt besprochenen Wendungen, wo eine Person und eine Sache mit Rücksicht auf ein Tun oder auf einen Zustand oder eine Eigenschaft in enger Beziehung zu einander stehend vorgeführt werden, fand größere Verbreitung in dem Fall, daß dem Sprechenden der Begriff der Person im Vordergrund stand. Die Person konnte so zum Mittelpunkt der Aussage, zum Subjekt gemacht werden. Für unser modernes Sprachgefühl gingen dabei die Griechen und Römer mit dem vom Verbum abhängigen Akkusativ über das hinaus, was wir Modernen diesem Kasus in Verbindung mit gleichartigen Verbalformen zuzumuten pflegen, z. B. mit Aus-

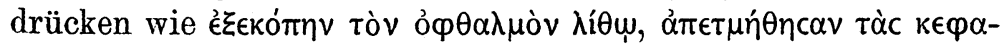

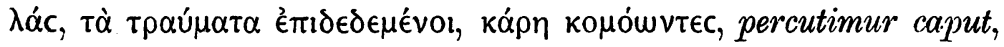
percussus pectus. Natürlich hatte man aber im Altertum überall auch die Möglichkeit eines Ausdrucks, bei dem nicht die Person,

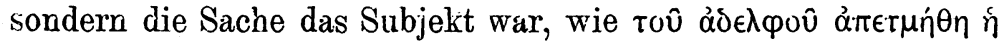

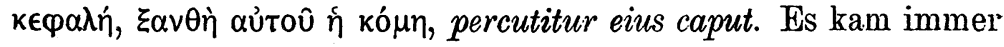
auf den ganzen Zusammenhang an, der bald die eine, die Person 
in den Vordergrund schiebende, bald die andere, die Sache in den Vordergrund bringende Wendung hervorrief. Vgl. z. B. Andok. De myst. 74, wo es erst mit sachlichem Subjekt heißt tî́c $\mu \grave{v} v$

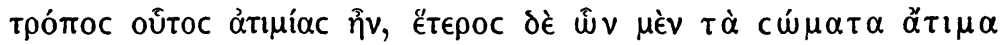

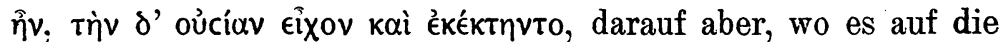

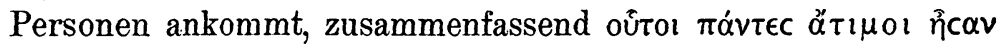
$\tau \grave{\alpha} c \omega ́ \mu \alpha \tau \alpha$.

Nun gab es neben dieser persönlichen Konstruktion mit dem Akkusativ der Sache ohne erheblichen Bedeutungsunterschied eine persönliche Konstruktion mit dem Instrumental der Sache. Der Instrumental hat in diesem Fall in der Grammatik denselben Beinamen wie der Akkusativ: Instrumental der Beziehung (Delbrück Grundriß 3, 272ff.). Ausgegangen mag er sein von Wendungen, in denen wir mit gebrauchen, wie 'hinken (hinkend) mit dem rechten Fuß', 'schnell mit den Füßen', 'ein Mann, Müller mit Namen’. Seine Verbreitung in den idg. Sprachen ist viel größer als die des Akkusativs der Beziehung, und man darf ihn getrost als uridg. Erbe ansprechen. Z. B. ai. nāragēna vihūrchati 'er hat nicht Schaden an einem Körperteil', rüpēna vikrtạ 'an Gestalt entstellt, häßlich von Gestalt', pädēna khänjah 'auf einem Fuße lahm', nä̀nnā 'mit Namen, Namens', griech. Xen. icxúeıv

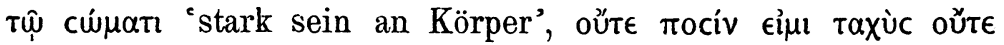

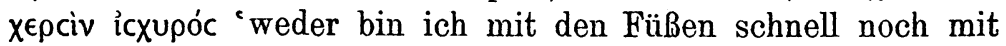

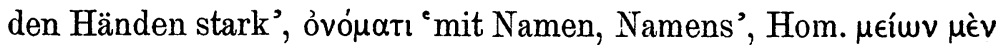

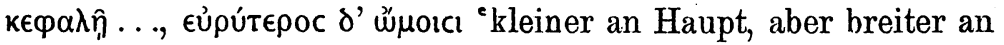
Schultern', lat. pedibus mobilis; lingua haesitans; nescio ut moribus sient vostrae; eunuchus nomine Pothinus, aisl. blindr bádum augum

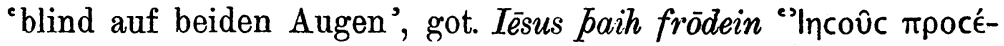

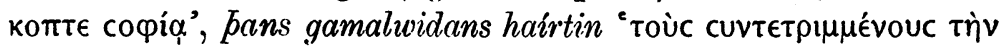

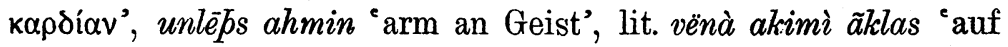
einem Auge blind', linksmas szirdże 'von Herzen froh', aksl. nemoštznz nogama 'schwach auf den Füßen', russ. dobr dušoju 'seelengut'. Deutlich ist, daß diese instrumentalische Ausdrucksweise die altertümlichere gewesen ist. Im Griechischen wurde sie schon seit vorhistorischer Zeit durch den Gebrauch des Akkusativs erheblich eingeschränkt.

Beide Ausdrucksweisen geben der Person die Subjektstellung und lassen die Sache als den untergeordneten Begriff erscheinen. Die jüngere Ausdrucksform, die mit dem Akkusativ, kann also nicht daher gekommen sein, daß man eine neue Aus- 
drucksweise zur Deckung des Bedürfnisses nach einer persönlichen Konstruktion zu schaffen gehabt hätte. Sie kann aber auch nicht etwa infolge davon entsprungen sein, daß sie den Vorzug größerer Deutlichkeit gehabt hätte. Diesen Vorzug dürfte man höchstens den noch jüngeren präpositionalen Umschreibungen zusprechen, die, schon in älteren Zeiten nicht ganz fehlend

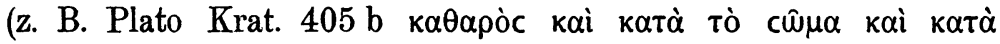
Tìv $\psi u x \eta ́ v)$, an mehreren Stellen des indogermanischen Sprachgebiets sowohl dem Instrumental der Beziehung als auch dem Akkusativ der Beziehung mehr und mehr Konkurrenz gemacht haben.

Das Verhältnis des Akkusativs der Beziehung zum Instrumental der Beziehung bestimmt sich vielmehr dahin: Die letztere Konstruktion war als eine Ausdrucksform mit der Person in Subjektstellung von Anfang an gegeben und dem Sprechenden geläufig. Die Akkusativkonstruktion dagegen und ihre Ausbreitung sind speziell dadurch veranlaßt worden, daß zwei Satzvorstellungen, eine mit einer Person in Subjektstellung und eine mit einer enge zur Person gehörigen Sache in Subjektstellung, welche zusammen Glieder einer weiteren Gesamtvorstellung waren, in éinen einfachen Satz zusammenzuziehen waren, ohne daß dabei etwas anderes erreicht werden sollte, als grammatische Unterordnung des Sachbegriffs unter den Personbegriff.

Die Ausbreitung des Beziehungsakkusativs ging, wie wir sahen, namentlich in der Richtung vor sich, daß er sich Partizipien und Adjektiven zugesellte. Waren nun z. B. die beiden

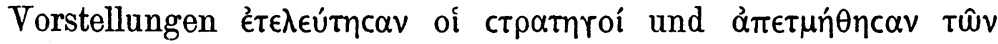

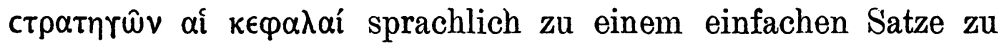
verschmelzen und hatte dabei der erste Satz, in dem oi ctpatnroí Subjekt war, für die Formung des ganzen Satzes die Führung, so war die einfachste Art der Einverleibung des zweiten Satzes

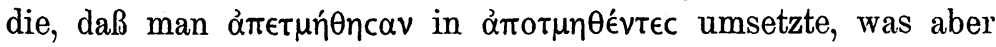

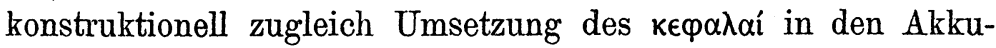
sativ erheischte. Ebenso ergab ein Satz mit Mєvé $\lambda \alpha o c$ als Subjekt,

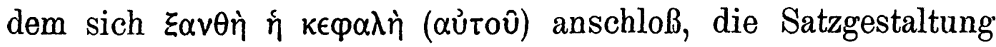

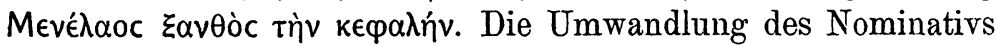
in den Akkusativ war somit nichts anderes ais die Überführung des Nomens ron der Herrscherposition im Satz zur Position des Beherrschten. 
6.

Dies bedarf mit Rücksicht auf die schon erwähnten Verbindungen eines nominalen Wortes mit einem Beziehungsakkusativ und auf die weiter unten zu nennenden gleichartigen Verbindungen noch einer weiteren Erläuterung. Ich muß dabei etwas weiter ausholen.

Auch noch in den Zeiten des bereits ausgebildeteren Satzes, der ein komplizierteres syntaktisches Gefüge darstellt, und so auch heute noch erscheinen vielfach, und zwar nicht bloß in der Sprache des niederen Volks, entweder Nominative für sich allein oder Nominative zusammen mit einem zu ihnen im Prädikatsverhältnis stehenden Nominativ, welche, äußerlich und rom Standpunkt des ausgebildeteren Satzes aus betrachtet, eine selbständige Äußerung darstellen, aber innerlich doch in engster Verbindung mit einem andern Satze oder einem Glied dieses Satzes stehen.

Es tritt also zunächst der Nominativ für sich allein auf nicht bloß z. B. in Überschriften oder bei Aufzählung von Gegenständen in einem Verzeichnis oder in pathetischer Schilderung, in Wortgemälden (wie Cic. Sest. 34, 74 clamor senatus, querellae, preces, socer ad pedes abiectus), in welchen Fällen der Gesichtspunkt innerlicher Über- und Unterordnung keinerlei Rolle spielt, sondern der Nominativ erscheint auch, in einen Satz eingefügt oder ihm angefügt oder vorausgestellt, als Vertreter eines nicht nominativischen Gliedes dieses Satzes. So z. B. nhd. von N. N., ordentlicher professor in Kiel, ist das und das veröffentlicht worden; dem herzog von Cumberland, königliche hoheit; mit meister Jean Prevost, ein guter maler; er zog nach der Normandie, eine treue und ergebene Provinz (s. ErdmannMensing Grundz. der deutsch. Synt. 1, 64f. 2, 115 f., Wunderlich Der deutsche Satzbau $2^{2}, 18 \mathrm{ff}$.). ${ }^{1}$ ). Aus attischen Inschriften werden bei Meisterhans-Schwyzer Gramm. der att. Inschr. ${ }^{3} 203 \mathrm{f}$. als solche 'unkonstruierte' Nominative angeführt $\pi \rho \omega \hat{\tau}$ oc $\rho u \mu o ́ c$

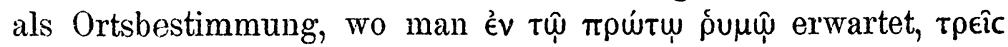

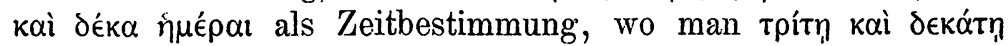

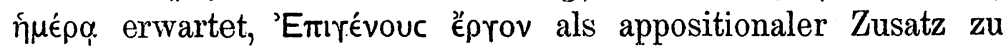

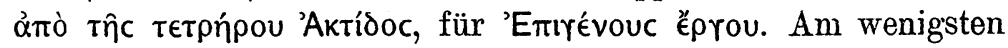
fällt dieser Nominativ auf, wenn er auf den Inhalt eines ganzen

1) $\mathrm{Ob}$ und wie weit unsere Sprachmeister diesen Gebrauch heute anerkennen oder nicht, ist hier natürlich völlig gleichgültig. 
Satzes sich bezieht, wie Otfr. 2, 9, 61f. thaz scāf gifiang er thō sār, wehsal gimuati bi sines sunes guati 'er nahm das Schaf, ein willkommener Tausch für seinen guten Sohn' (Erdmann-Mensing

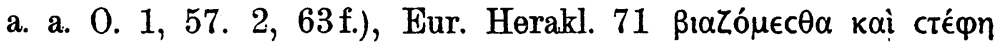

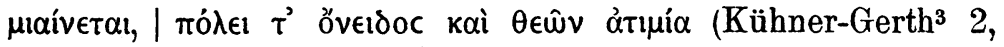
1, 284 f.), Cic. Tusc. 1, 26, 65 nec Homerum audio, qui Ganymeden $a b$ dis raptum ait propter formam, ut Jovi bibere ministraret, non iusta causa, cur Laomedonti tanta fieret iniuria (Draeger Hist. Synt. ${ }^{2}$ 1, $669 \mathrm{ff}$.). Denn hier kommt eine eigentliche Einverleibung in einen Satz für den Nominativ nicht in Frage. Die relative Selbständigkeit tritt dann besonders zutage, wenn ein solcher Nominativ voransteht, wie Otfr. 4, 4, 32 seltsāni rächa, breittun iro lachan 'eine seltsame Begebenheit, sie breiteten ihre Kleider aus'1). So können nun auch Nominative, die zusammen mit einem nominalen Prädikat zunächst einen Satz für sich ausmachen, in so enger innerer Beziehung zu einem andern Satz stehen, daß sie wie Glieder dieses Satzes empfunden werden. Darauf beruht z. B. der dem Genitivus absolutus des Griechischen und andern ähnlichen absoluten Partizipialkonstruktionen der indogermanischen Sprachen gegenüberstehende 'Nominativus absolutus' des Gotischen: Mark. 6, 21 jah waürbans dags gatils, pan Hērōdis mēla gabaúrpais seinaizōs nahtamat

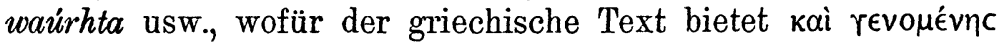

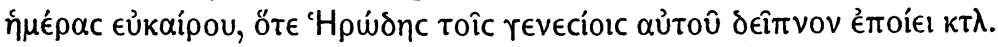
(J. Grimm D. G. 4, 895, Streitberg Got. Elementarb.2 155. 209)²).

1) Statt des Nominativs tritt bei solchen Appositionen im Griechi-

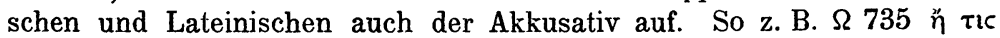

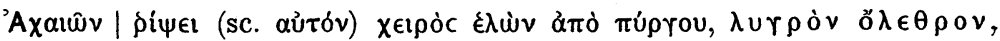

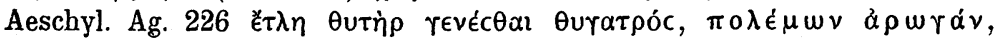
Cic. de or. 2, 19, 79 quinque faciunt membra, rem non difficilem, Liv. 5, 47, 8 selibras farris contulerunt, rem dictu parvam. S. Kühner-Gerth ${ }^{3}$ 2, 1, 284 f., Nachmanson Eranos 9, 46 ff., Draeger Hist. Synt. ${ }^{2} 1,669 \mathrm{ff}$., C. F. W. Müller Synt. des Nom. und Akk. 161. Offenbar hat hier zu der Zeit, wo dieser Akkusativgebrauch aufkam, das Substantivum als Objekt zu einem transitiven Verbum vorgeschwebt. Es gehört das also in derselben Art ins Gebiet der. Verbalellipse, wie z. B. $\gamma v \dot{u} \mu \eta \nu \varepsilon^{2} \mu \eta \dot{v} v$ bei Aristoph.

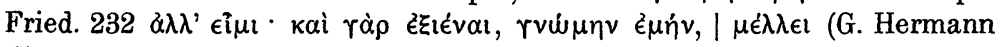
Vig. Idiot. Gr. p. 883), oder wie wenn bei uns eine Gastwirtschaft plakatweise ankündigt Kः ${ }^{*}$ ätigen Mittagstisch, Reinen Apfelwein u. dgl. (dergleichen Ankündigungen erscheinen daneben auch in nominativischer Form).

2) Es ist dies einer der verschiedenen Versuche des Wulfila, die dem Gotischen fremde absolute Partizipialkonstruktion nachzuahmen (vgl. 
In allen diesen Formen macht das Wort in Nominativform, wenn man die in den Schriftsprachen üblich gewordenen verwickelteren Satzgestaltungen zum Maßstab nimmt, den Eindruck von unverarbeitetem Rohmaterial. Man vergleiche in dieser Beziehung den Anfang desjenigen Berichts, der im Beginn der 1. Herakleischen Tafel über Vermessung von heiligem Gelände erstattet wird, mit dem Anfang des mit Zeile 95 beginnenden $\mathrm{Be}-$

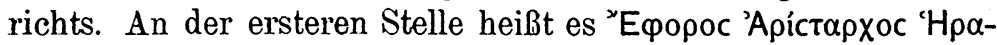

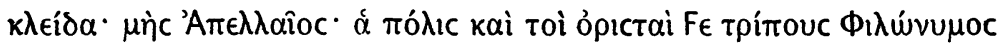
Zwmupíckw usw.: nur Nominative (mit allernächstem Zubehör) aneinander gereiht, aber keine alle Einzelvorstellungen zu einem Ganzen verbindende Satzbildung; diese hebt erst mit den Worten

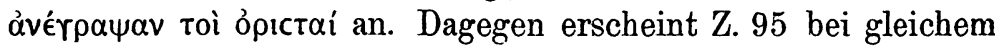
Inhalt ein Satz, der dem simultanen Erfassen der ganzen Vor-

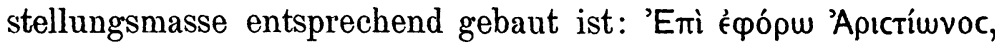

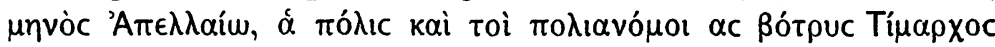

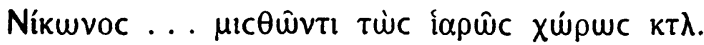

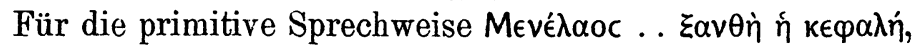
woraus durch syntaktische Einverleibung des zweiten Teils Mevé-

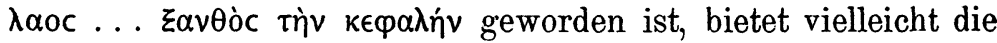
beste Parallele im Kreis der idg. Sprachen eine in der russischen Volkssprache übliche Ausdrucksweise, über die kürzlich Leskien IF. 23, $204 \mathrm{ff}$. gehandelt hat. Es heißt da z. B. Nastasja zototaja kosa d. i. wörtlich 'Anastasia golden das Haar' = 'Anastasia mit goldenem Haar, die goldhaarige Anastasia', carica zototyja kudri 'die Kaiserin golden die Tocken' = 'die Kaiserin mit goldenen Locken, die goldlockige Kaiserin'. Daß das Adjektiv von Haus aus in einem prädikativen Verhältnis zu dem den Teil bezeichnenden Substantiv stehend empfunden war, ergibt sich daraus, daß z. B. der Gedanke 'da begegnet ihm eine dickbäuchige Alte' auch so ausgedrückt erscheint: povstrěčajet jemu starucha totstoje jejo brucho d. i. 'eine Alte, dick ihr Bauch, crassus eius venter'. Zum Teil muß dieser zweigliedrige Zusatz zu dem die Person bezeichnenden Substantiv wie eine Art von Kompositum empfunden

Delbrück Grundriß 4, 495 f., Streitberg a. a. 0.). Dabei griff Wulfila zu einer gewiß echt volkstümlichen Ausdrucksweise. Warum der überlieferte Ausdruck mit dem syntaktischen Charakter des Nominativs nicht vereinbar sein soll, wie van Helten PBrB. 35, 310f. sagt, vermag ich nicht einzusehen. Van Heltens Vorschlag, die Überlieferung in jah pan warb dags gatils zu korrigieren, ist abzuweisen. 


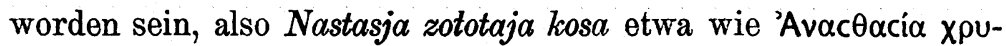
có $\theta$ plz oder Anastasia goldhaar. Das zeigt der Umstand, daß, wenn es sich um Casus obliqui handelt, der attributive Zusatz in kasueller Kongruenz mit dem Personennamen vorkommt, wie syn caricy zototoj kosy 'Sohn der Kaiserin, der goldhaarigen'. Doch kann der Zusatz auch die Nominativform beibehalten, wahrt sich also auf diese Weise eine gewisse syntaktische Selbständigkeit, wie op'at' sdétataś cariceju (Instr. Sing.) zototyja kudri (Nom. Plur.), 'sie wurde wieder zur Kaiserin, golden die Locken' = 'zur goldlockigen Kaiserin'.

In dieser sehr primitiv aussehenden Ausdrucksweise ist zugleich, wie im Eingang des Leskienschen Aufsatzes wenigstens angedeutet ist, eine Vorstufe zur Entstehung der exozentrischen Nominalkomposita wie úkúnouc, $\lambda \epsilon u \kappa \omega ́ \lambda \epsilon v o c$ zu sehen. Womit natürlich nicht gesagt sein soll, daß der russische Ausdruck in ununterbrochener Kontinuität aus jener Zeit der idg. Urgemeinschaft ererbt sei, in der solche Komposita zuerst aufkamen; denn der Typus konnte sich im Lauf der Sprachgeschichte immer von neuem erzeugen. Diese exozentrischen Komposita werden nun

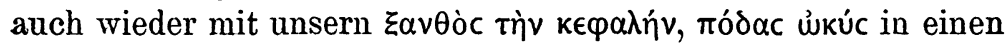
näheren historischen Zusammenhang gebracht von Neckel IF. 19, 253, und so muß ich auch auf diese Komposita hier eingehen.

Nach dem Vorgang von Justi und Jacobi nimmt Neckel an, daß Bildungen wie ai. grivvabaddhá- 'mit gebundenem Hals',

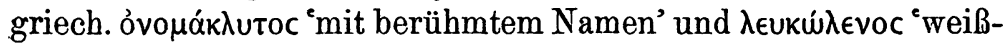
armig' hervorgegangen seien aus Sätzen, die, als eine Art von Nebensätzen, anderm untergeordnet waren. Ursprünglich, und zwar in der vorflexivischen Periode, sei danach der Sinn von grivabaddhá- gewesen '(der) Hals gebunden', der Sinn von $\lambda \in U-$ $\kappa \omega ́ \lambda \in v o c$ 'weiß (der) Arm'. In einer Äußerung nun wie 'Mann - (der) Hals gebunáen - wurde hingeführt' habe man 'Mann' mit solcher Entschiedenheit zum Mittelpunkt der ganzen Aussage und somit zum Subjekt des Ganzen gemacht, daß 'Hals' dem gegenüber zurückgetreten sei und an den Kennzeichen des Subjekts nicht teil bekommen habe; so sei das Stammkompositum 'halsgebunden' entstanden. Weiter heißt es dann: "Die unsern Fällen nächstverwandte Art der Differenzierung des Doppelsubjekts ist der sogeu. griechische Akkusativ, z. B. in hom. ßonेv óra $\alpha$ óc. Dieser Akkusativ scheint ausgegangen von solchen Neutra, bei denen der Objektkasus mit dem reinen Stamm identisch war, 


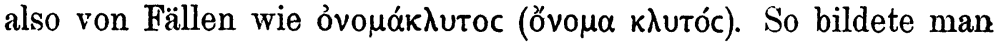

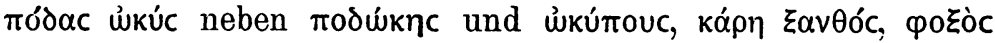

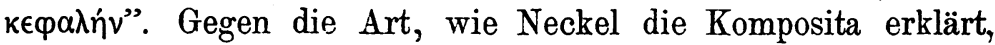
habe ich nichts einzuwenden. Und auch bezüglich der Entstehung

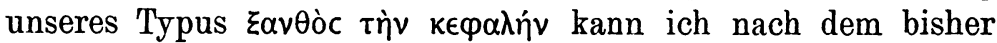
Dargelegten im Prinzip nur durchaus beistimmen. Nur hat Neckel nicht erkannt, wie man gerade zum Akkusativ gelangt ist. Denn daß die äußere Gleichheit der Stammform mit der Form des Nom.Akk. Neutr. den Anstoß zu der ganzen Bewegung gegeben habe,

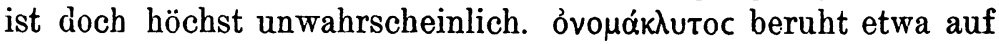

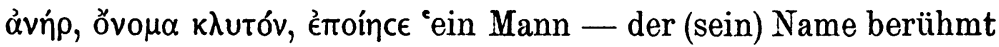

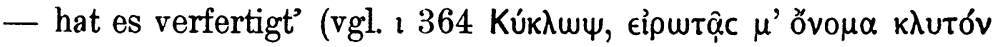

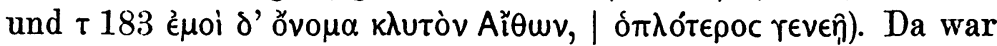

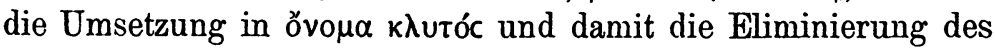

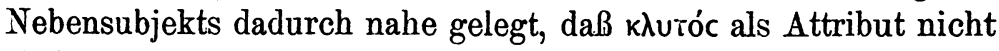
bloß von ơvoua, sondern auch von Personen im Gebrauch war ${ }^{1}$ ). ǒvo $\mu$ aber als Akkusativ war, wie wir gesehen haben, begründet

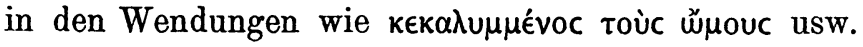

Neckel fährt fort, kommen gleichartig mit aisl. halsdigr 'mit dickem Hals', halslangr 'mit langem Hals' und ähnl. und fügt hinzu, es sei denkbar, daß in diesen aisl. Bildungen ebenfalls der Akkusativ steckte. Dies ist in der Tat sehr wohl möglich, und besonders das Partizip fótbrotinn 'wer einen gebrochenen Fuß hat, mit gebrochenem Fuß' (zu brióta 'zerbrechen') macht es wahrscheinlich, daß das erste Glied der Zusammensetzung ebenso $\mathrm{zu}$ beurteilen ist wie das Vorderglied in halshoggua $\mathrm{u}$. dgl. (S. 128). Die Formen wären mit den alten Stammkomposita allmählich ganz zusammengefallen.

\section{7.}

Ich wende mich nun zu den deutschen Partizipialkonstruktionen wie: den kopf entblößt, trat er ein; er fleht, die hände zu Zeus erhoben, deren Akkusativ, wie S. 123 bemerkt ist, von Ziemer und Cauer mit dem griechischen Akkusativ ver-

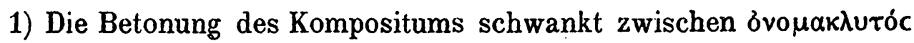

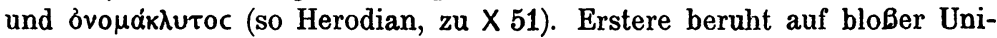

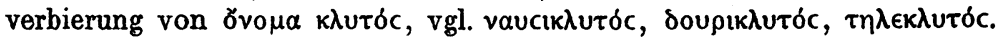

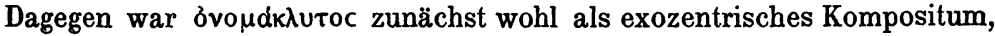

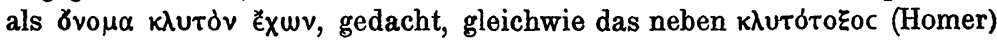

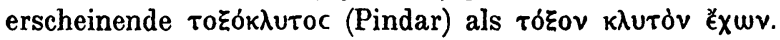


glichen worden ist, um diesen dem Verständnis des Anfängers näher zu bringen. Vgl. Grimm D. G. Neudr. 4, 1096 ff., Andresen Sprachgebr. u. Sprachricht. ${ }^{9} 176 \mathrm{ff}$. , Erdmann-Mensing Grundz. 1, 58. 60 f., Wunderlich Der deutsche Satzbau 1², $395 \mathrm{ff}$.

Man hält diese Ausdrucksweise, so viel ich weiß, allgemein für einen Romanismus oder zugleich auch Latinismus, und das wird richtig sein. Der Kasus des Substantivs war in der fremden Sprache formal indifferent: an sich konnte er von den Deutschen ebenso gut als Akkusativ wie als Nominativ angesehen werden, z. B. franz. la danse finie on se met $\grave{a}$ table. Daß der Deutsche den Akkusativ wählte, war auch hier wieder zunächst durch die notwendige Unterordnung unter das Hauptverbum nebst dessen nächstem Zubehör veranlaßt.

So weit ich das einschlägige Material überschaue, haben wir für den uns hier angehenden syntaktischen Gesichtspunkt zwei Gruppen von Beispielen zu unterscheiden. Erstens die Fälle, wo sich das Partizipium wie ein Participium coniunctum zum Subjekt des Hauptverbums ziehen läßt, wie in er fleht, die hände zu Zeus erhoben. Zweitens die Fälle, wo das nicht möglich ist, wie: gesetzt den fall, daß es regnet, (so) wird der ausflug unterlassen; das buch kostet 10 mark, den einband mitgerechnet; gehört des volkes laute klage, gefiel es, einen landtag auszuschreiben; das geschehen, hänge die entscheidung von dem könige selber $a b$ (s. Grimm und Andresen a. a. 0.).

Daß im ersteren Fall das Substantiv jm Akkusativ häufig, ja meistens als abhängig vom Partizipium empfunden wird, ist mir unzweifelhaft. Und das wird durch assoziativen Einfluß von zweierlei Konstruktionen hervorgerufen sein, die der Deutsche schon besaß, ehe die romanische Partizipialkonstruktion Anklang fand. Erstlich durch die Wendungen wie auf den mauern erschienen, den säugling im arme, die mütter; er eilte herbei, tränen im auge; die hände auf dem rücken, stand er da, wo von altersher ein habend vorgeschwebt hatte (vgl. IF. 18, $127 \mathrm{ff}$.). Zweitens und hauptsächlich aber durch den Akkusativ der Sache, welchen die von alter Zeit her im Aktiv mit dem doppelten Akkusativ korstruierten Verba lehren usw. in der persönlichen passivischen Wendung beibehalten hatten, und den sie mithin auch beim Passivpartizip hatten, wenn dies ohne sein oder werden gebraucht war, wie mhd. (die vrouwe) zuht gelëret (oben S. 126f., Grimm a. a. $0.729 \mathrm{f} .757 \mathrm{f}$.). 
Hier hätten wir es denn mit einem Gebrauch des Akkusativs im Deutschen zu tun, auf den der Name Akkusativ der Beziehung anwendbar wäre, und der Fall wäre ähnlich dem, der uns S. $132 \mathrm{ff}$. im Lateinischen begegnete, der darin bestand, daß das durch die griechische Sprache gegebene Vorbild eine Befruchtung und Erweiterung eines einheimischen Akkusativgebrauchs hervorrief. Ein erheblicherer Unterschied gegen den Vorgang in der lateinischen Sprache wäre nur der, daß bei uns der Akkusativ sich von den Partizipien nur ausnahmsweise auf die eigentlichen Adjektiva verpflanzt hat, wie etwa den kopf frei von sorgen, reiste er $a b^{1}$ ).

Abgesehen von Wendungen wie den fall gesetzt, $d a \beta \ldots$ (auch bloß gesetzt, daß . . .) und solchen, in denen der Akkusativ das Neutrum eines Pronomens ist, wie dies abgerechnet, das ausgenommen, ist die in Rede stehende Partizipialkonstruktion nur noch dann geläufig, wenn der Urheber der durch das passive Partizip ausgedrückten Handlung zugleich Subjekt des regierenden Verbums ist, wie in er fleht, die hände zu Zeus erhoben. Man bildet also jetzt kaum mehr Sätze wie Chamissos angeführten Satz gehört des volkes laute klage, gefiel es, einen landtag auszuschreiben. Diese Tatsache darf als Bestätigung dafür gelten, daß das Partizipium für uns nicht oder wenigstens nicht bloß Attribut zu dem Akkusativ ist. Jedenfalls ist der von C. F. W. Müller Syntax des Nom. und Akk. im Lat. S. 128 Fußn. 1 gegen Cauer erhobene Einwand hinfällig.

Übrigens bedürfen diese Partizipialkonstruktionen des Neuhochdeutschen hinsichtlich ihres Entwicklungswegs auf Grund der Sprachdenkmäler einer noch genaueren Untersuchung als sie bis jetzt nach meinem Wissen erfahren haben, und so gebe ich meine Auffassung nur unter Vorbehalt.

8.

Als 'Akkusativ der Beziehung' stellt sich weiterhin griech.

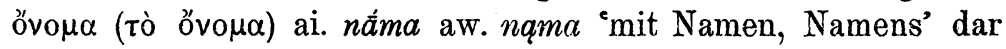
(vgl. die S. 124 genannte Literatur) ${ }^{2}$ ).

1) Virg. Aen. 1, 320 dederatque comam diffundere ventis, | nuda genu nodoque sinus collecta fuentes wird von Voss übersetzt: nackend das knie und im knoten die fliessenden schöße gesammelt.

2) Vom Altpersischen sehe ich ab, weil die Lesung des dem aw. nama entsprechenden Wortes Schwierigkeiten bereitet, die noch ungelöst 
Die altertümlichsten Wendungen sind nach meinem Dafür-

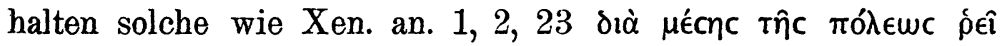

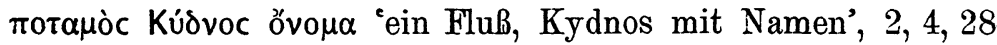

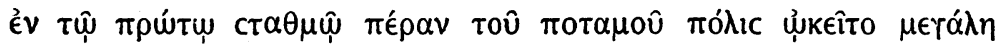

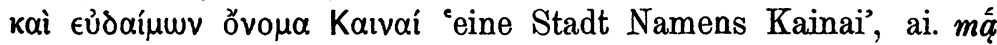
dhur índra náma dēvátā diváš ca gmáš cāpá ca jantávah 'mich, den Indra mit Namen (mit dem Namen Indra), haben als Gott eingesetzt die Geschöpfe des Hinmels, der Erde und der Wasser, yád nibarháyō námucị náma māyinam 'als du niederstießest den Dämon mit Namen Namuci', páršur ha näma mānaví sāká sasūva vịsatim 'die Manutochter, Paršu mit Namen, hat zwanzig auf einmal geboren', aw. V. 7, 16 ar`dvi nama āpa 'das Ardvī genannte Wasser'. Ursprünglich hatten der Eigenname und das Wort name, im Verbältnis von Subjekt und Prädikat zu einander stehend, einen der Form nach selbständigen Satz gebildet: $\rho \in \hat{\imath}$

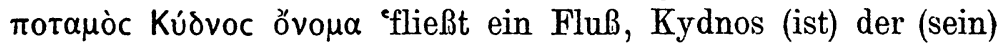
Name'. Passend verweist Gray auf Beowulf 1457 woes póm hoeftméce Hruntinz nama, d. i. wörtlich 'es war ihm ein Heftschwert, Hrunting (war) der Name' (vgl. Foy S. 178). Die Umwertung von name zum Akkusativ erfolgte entsprechend der Änderung von

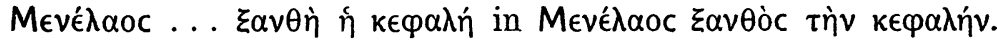
Die Benennung, zu der das Wort name gehörte, konnte auch ein Adjektiv sein, wie Yt. 15, 46 taxmō nąma 'stark der Name', $=$ 'stark genannt'.

Bei mạ ... indra náma und námucị náma māyínam darf man daran denken, daß sie direkt den Typus fortsetzen, bei dem náma noch nicht dem Eigennamen als 'Akkusativ der Beziehung' untergeordnet gewesen ist. Es läßt sich annehmen, daß die alte nominativische Verbindung indrō náma 'Indra (ist) der Name', námucir náma 'Namuci (ist) der Name' direkt durch Kasusassimilation in den Akkusativ umgesetzt worden sei, in derselben Weise, wie im Russischen najd'oš svoju mat' zototaja kosa ('du wirst finden deine Mutter, golden das [ihr] Haar') zu svoju mat' zototuju kosu 'deine Mutter die goldhaarige' geworden ist (S. 139f.). In der Zeit, aus der uns solche indische Sätze überliefert sind, wäre aber náma jedenfalls, nach Analogie seines sonstigen Gebrauchs, umempfunden, also als abhängig vom Eigennamen empfunden gewesen.

sind, und die auch ich nicht zu erledigen weiß (vgl. Foy IF. 12, 172, Bartholomae Altiran. Wtb. Sp. $1063 \mathrm{f}$.). 
Daß das aus dem Eigennamen und dem Akkusativ name bestehende Satzstück, das appositioneller Zusatz zu einem appellativischen Substantiv war, auch vor dieses Substantiv gesetzt erscheint, darf nicht auffallen. Auch sonst können Appositionen dem Substantiv, das durch sie näher bestimmt wird, vorausgeschickt werden (Delbrück Grundriß 5, 198f.). Speziell für unsern Fall vergleiche man das von Leskien S. 204 angeführte krasnaja krasa cornaja kosa car'-dèvica, d. i. 'rot die Gesichtsfarbe, schwarz das Haar, Kaiser-Jungfrau' = 'die im Gesicht rotfarbige, schwarzhaarige Kaiser-Jungfrau’.

Die Umwertung des Nominativs name zum Akkusativ, durch die dieses Wort gewissermaßen zum bloßen Exponent der Funktion des Eigennamens als solchen gemacht wurde, hatte zur Folge, daß man diesen Akkusativ auch dann dem Eigennamen beigab, wenn dieser nicht Apposition eines Appellativums war. Oder anders ausgedrückt: der aus dem Eigennamen und dem Beziehungsakkusativ bestehende appositionelle Zusatz zu einem Appellativum trat für das Appellativum selbst ein. Das

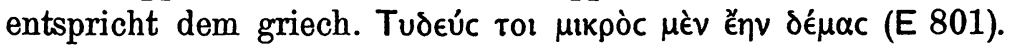
So ai. asāu namāham asmi 'der und der (der N. N.) mit Namen bin ich' (woran sich anschloß kó námāsi 'wer mit Namen bist

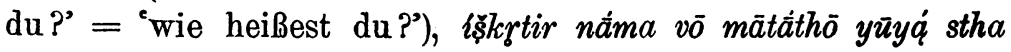
niṣ̌krtīh 'Heilung mit Namen ist (Heilung ist benannt) eure Mutter, darum seid auch ihr Heilungen', aw. vairiš yō haosravá nąma 'der See, welcher H. mit Namen ist (H. heißt)', vayuš bā nquma $a h m i$ 'die Atmosphäre mit Namen bin ich (heiße ich)', Hesiod

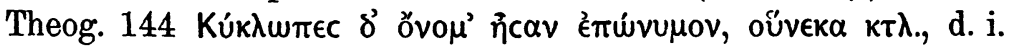
'Rundaugen waren sie mit ihrem Zunamen' = 'waren sie zubenannt'. Diese Anhängung von name konnte um so leichter Platz greifen, als Eigennamenwörter und Appellativwörter in diesen alten Zeiten sehr oft nicht ohne weiteres zu unterscheiden waren, wie ja auch noch heute, wenn jemand z. B. sagt ich bin Müller, dies zweideutig sein kann.

Im Grunde ist die hier vorgetragene Erklärung des Akkusativs náma nąma ǒvoua schon von Gray IF. 11, $307 \mathrm{ff}$. gegeben, und Foy in seiner - zum großen Teil durchaus berechtigten - Polemik gegen Gray weist sie wenigstens nicht von der Hand (IF. 12, 178). Anders erklärt unsern 'Akkusativ der Beziehung' Delbrück Grundriß 3, $387 \mathrm{f}$. Er geht aus von Sätzen wie aw. spānom yim mašyāka avi dužvačarhō dužakom nąma aojaite 'den Hund, den 
die übelredenden Leute Dužaka mit Namen nennen' und Hom.

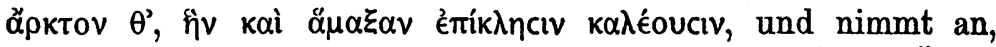
daß der Sinn der Beziehung sich von da aus durch eine Übertragung entwickelt habe. In dem aw. Satz sei nąma ursprünglich wohl Apposition gewesen: "welchen die Leute Dužaka als Namen nennen'. Ob auch é $\pi i k \lambda \eta c i v$ als Apposition aufzufassen oder als Akkusativ des Inhalts nahe zu kaגeîv zu ziehen sei (so auch

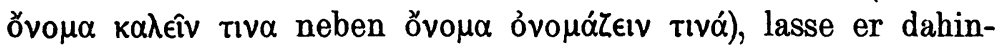
gestellt. Der neue Typus nun scheine entstanden zu sein, indem

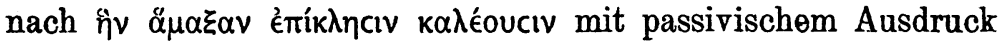

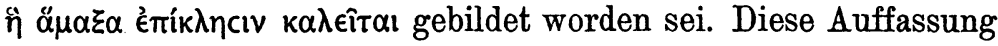
ist mir unannehmbar, weil sie uns nötigen würde, die Entstehung des Beziehungsakkusativs ai. náma usw. unter einen ganz andern Gesichtspunkt zu bringen als die der oben behandelten Fälle und namentlich auch die der unten noch zu besprechenden Beziehungsakkusative aw. masō, fra $\theta \bar{o} \mathrm{u}$. dgl. und griech. révoc,

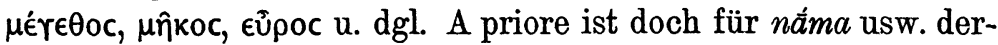
selbe Entwicklungsgang wie in allen diesen Fällen wahrscheinlich, um so wahrscheinlicher, als auch bei dem Worte name wieder dem Akkusativ 'der Beziehung' der Instrumental 'der Beziehung’ zur Seite steht, nämlich ai. námnā, griech. ỏvó $\mu \alpha \tau$ (entsprechend lat. nomine), wie wir denn auch weiter unten z. B. $\mu \in \gamma^{\prime} \in \in \in 1$ neben $\mu$ '́ $\gamma \in \theta o c$ 'an Größe' begegnen werden. Immer-

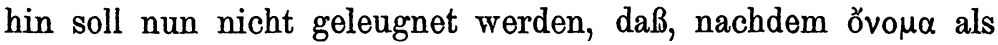
Akkusativ 'der Beziehung' bereits aufgekommen war, das als Akkusativ des inneren Objekts stehende övo $\mu \alpha$ bei der Aus-

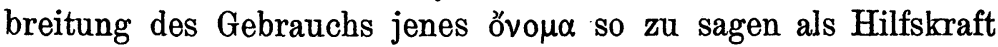
eine gewisse Rolle gespielt habe. Denn eine gewisse Assoziation der beiden ǒvoux mußte stattfinden; die Grenzen, die wir Grammatiker durch Gebrauchseinteilungen und Anwendung verschiedener Benennung anbringen, bestehen ja für den Sprecher selbst niemals in der Schärfe, in der wir sie ziehen.

9.

In ähnlicher Weise wie övo $\mu \alpha$ sind bei den Griechen révoc und reveńv Akkusativ 'der Beziehung' geworden. Ursprünglich

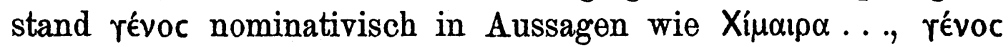
Өєîov, d. h. 'ảie Chimäre ..., der (ihr) Ursprung ein göttlicher'.

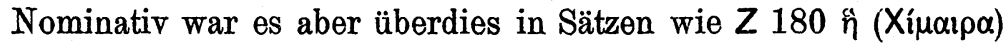

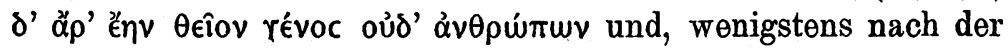




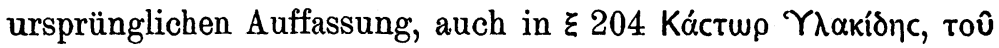

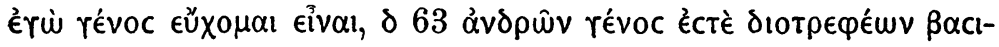
$\lambda \eta \dot{w v}$. In den letzteren Fällen vollzog sich die Umwertung: 'dessen der Herkunft nach zu sein ich mich rühme, zu dem der Herkunft nach zu gehören ich mich rühme'. Daher denn auch mit

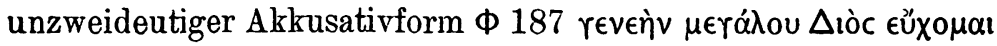

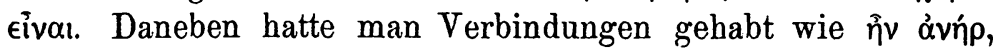

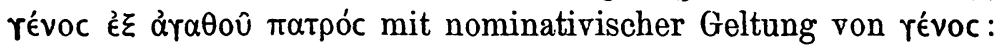
'es war ein Mann, die (seine) Herkunft von einem tüchtigen Vater', und dann wurde révoc auch hier akkusativisch: $\equiv 113$

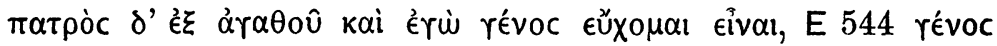

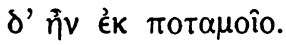

Hieran mag aw. $a r^{2} j o ̄$ (Akk. Sing. Neutr.) 'an Wert, um den Wert, Preis von ...' angeschlossen werden. Denn z. B. die Satzbildung F. $3 \mathrm{~h}$ aspō arryōtomō daŕhve var manō ašta gavąm azinq̨m $a r^{2} j \delta$, 'das edelste Roß aus der Auslese eines Landes gilt acht trächtige Kühe an Wert, hat den Wert von acht trächtigen Kühen',

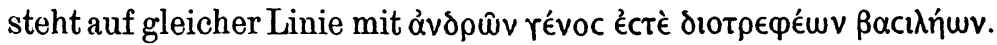

Endlich ist $\mathrm{zu}$ nennen die besondere Übereinstimmung zwischen Griechisch und Iranisch im akkusativischen Gebrauch von Substantiva, die 'Größe', 'Länge', 'Breite' u. dgl. bezeichnen.

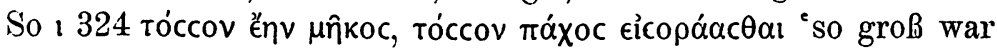
sie (die Keule) an Länge, so groß an Dicke von Ansehen', $\lambda 311$

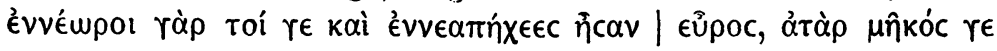

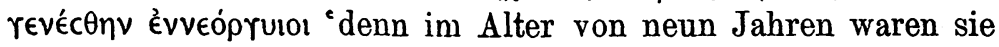
Menschen von neun Ellen in der Breite, in der Länge aber wurden

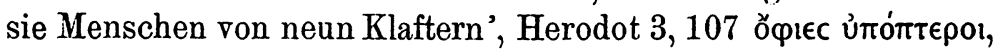

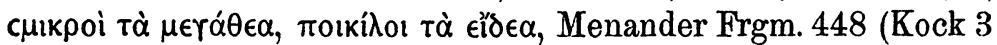

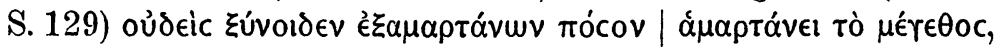

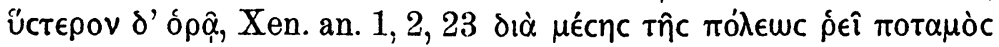

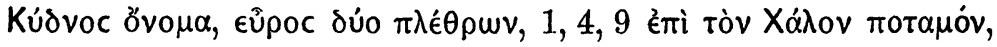

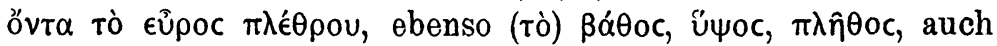

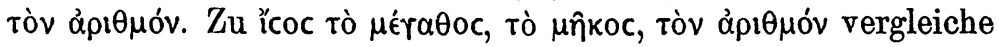

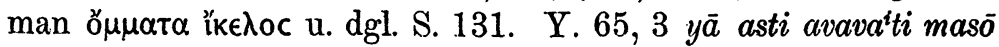

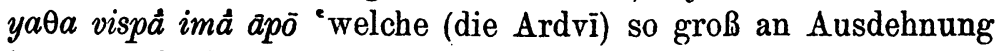
ist wie alle Gewässer', Y. 19, 7 astiča im zấ avaiti bązo yavaiti fräasčit 'die Erde ist ebenso groß an Tiefe wie an Breite', V. 3, 16 čvat drājō hača ... 'wie viel an Länge von ...' = 'wie weit weg von ..., V. 6, 1 čvantam drājōo zrvānom 'wie viel Zeit an Länge' = 'wie lange Zeit', V. 9, 3 hō par ${ }^{2} \theta w e$ a'́rhà zamo 


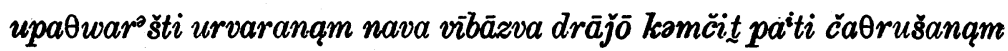
'er schneidet auf der Oberfläche der Erde Pflanzen ab neun Klafter an Länge nach jeder der vier Seiten". Auch hier, wo abermals der Akkusativ mit dem Instrumental der Beziehung wechselt (griech. $\mu \in \gamma^{\prime} \in \epsilon \in$ u. dgl.), ist nach dem oben Dargelegten von Aussageformen auszugehen, in denen die Substantiva $\mu \hat{n} \kappa o c$ masō usw. Nominativ waren. Den Satz Xen. an. 1, 2, 23 z. B. hat man sich, nach dem ursprünglichen Sinn solcher Aussageformen, zu denken als: 'durch die Stadt fließt ein Fluß, Kydnos (ist) der (sein) Name, die (seine) Breite (beträgt) zwei Plethren';

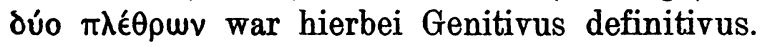

10.

Alle Beziehungsakkusative, die wir bis jetzt besprochen haben, brachten wir entwicklungsgeschichtlich unter einen einheitlichen Gesichtspunkt, unter den, daß sie unmittelbar oder mittelbar zurückgehen auf den Subjektskasus eines in engster Abhängigkeit von einem andern Satz befindlichen Satzes; dieser Subjektskasus mußte infolge der Einverleibung des betreffenden Satzes in einen größeren grammatischen Satzorganismus seine Stellung als Subjekt und damit die Nominativform aufgeben. Man hat nun den Namen Akkusativ der Beziehung auch noch auf einige anders geartete Gebrauchsweisen angewendet. Das wäre an sich nicht zu tadeln, weil der Name ja höchst dehnbar ist und im Grunde für die verschiedensten Anwendungsweisen der Akkusativform benutzbar wäre. Man wird aber gut tun, den 'Akkusativ der Beziehung' nicht zum Sammelbecken für alle Gebrauchsarten des Akkusativs zu machen, die sich nicht glatt unter den bereits etikettierten andern Anwendungsweisen dieses Kasus unterbringen lassen, vielmehr den Namen, falls man ihn überhaupt beibehalten will, auf die von mir oben besprochenen Gebrauchsarten zu beschränken. Dreierlei habe ich hier zu erwähnen :

1. Scheftelowitz ZDMG. 57, 159 bringt unter der Überschrift Akkusativ der Beziehung (accus. graec.) aus dem Awesta die beiden Stellen Y. 50, 10 und V. 7, 51 bei. Daß sie aber beide mit unserm Akkusativgebrauch, wie immer man ihn definieren mag, nichts zu schaffen haben, braucht nicht besonders bewiesen zu werden. Über die dritte von Scheftelowitz unter jene Überschrift gebrachte Stelle V. 18, 38 ist oben S. 128 gehandelt. 
2. Weiter muß ich jetzt abtrennen einen von mir selber Griech. Gramm. ${ }^{3}$ S. 382 an unsern 'Akkusativ der Beziehung' angeschlossenen Akkusativgebrauch der griechischen Sprache, zu dessen Angliederung mich der Umstand verleitet hatte, daß die Benennung 'der Beziehung' an sich auf ihn vorzüglich paßt.

Bei der sogenannten Herübernahme des Subjekts des Nebensatzes als Objekt in den Hauptsatz, wie B 409 ᄁֶठ $\delta \epsilon \epsilon$ rà $\rho$

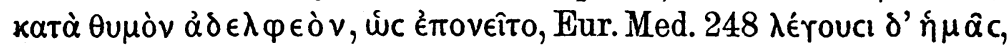

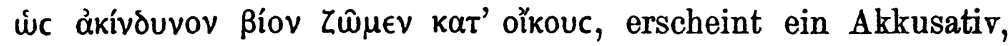
der von Haus aus unzweifelhaft der gewöhnliche Objektsakkusativ zum Verbum seines Satzes gewesen ist. Es änderte sich hier das Sprachgefühl mit der Zeit. Das zeigen ganz klar die Fälle, wo an der Stelle des zu erwartenden transitiven Verbums ein intransitiver oder passivischer Ausdruck auftritt, wie Aristoph.

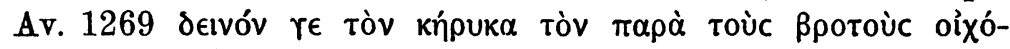

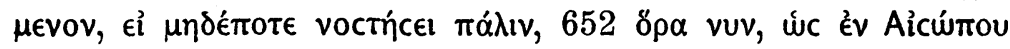

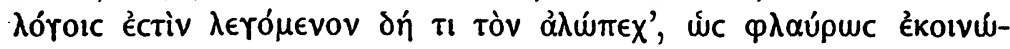

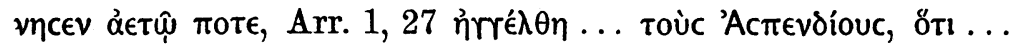

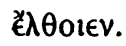

Daß das nun etwas ganz anderes ist, als was wir oben als 'Akkusatir der Beziehung' bezeichnet haben, braucht nicht näher ausgeführt zu werden. Ich glaube jetzt annehmen zu müssen, daß die Griechen diesen alten Objektsakkusativ, der zugleich das Subjekt des folgenden Nebensatzes war, in eine derartige engere grammatische Beziehung zu diesem Nebensatz brachten, daß sich sein Verhältnis zum Verbum des Hauptsatzes lockerte und verdunkelte - eine Art von Gliederungsverschiebung, die ihr nächstes Analogon in der Entstehung des 'Accusativus cum infinitivo' hat, dessen Subjekt bekanntlich ursprünglich ebenfalls Objekt des regierenden Verbums gewesen ist, von diesem aber sich abgelöst hat. Man mag auch die Entstehung unserer Nebensatzkonjunktion $d a \beta$ aus einem vom vorangehenden Verbum regierten das (ich sehe, daß er hinkt aus ich sehe das: er hinkt) vergleichen und anderes derart, was bei Paul Prinzipien $4282 \mathrm{ff}$. besprochen wird. Gleichwie also bei der Konstruktion des Accusativus cum infinitivo die anfängliche syntaktische Natur des Akkusativs damit definitiv als verschoben sich darstellt, daß

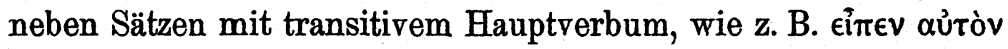
$\pi \alpha \rho \in \lambda \theta \epsilon \hat{v}$, solche mit nichttransitivem Hauptverbum auftreten, 


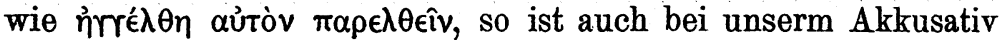
als dem Vorverkündiger des Subjekts des konjunktionalen Nebensatzes der Übergang von transitiven zu nichttransitiven Hauptverba das Kennzeichen der vollzogenen syntaktischen Umwertung. Und wenn nach Vollzug dieser Umwertung der Akkusativ die Stellung unmittelbar vor dem konjunktionalen Nebensatz, in der er die Änderung seiner syntaktischen Geltung erfahren hatte, nicht mehr festzuhalten brauchte - vgl. Xen. Kyr. 2, 1, 5 тo ̀̀ c

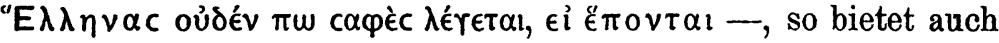
hier der Accusativus cum infinitivo eine Parallele in Satzge-

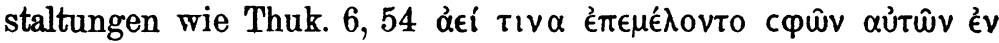

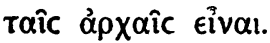

3. Reichelt Awest. Element. 229f. erwähnt unter der Überschrift 'Akkusativ der Beziehung' nicht nur die oben aus dem A westischen angeführten Fälle nąma 'mit Namen' (S. $143 \mathrm{ff}$.), $a r^{\curvearrowright} j \bar{o}$ 'an Wert' (S. 147), maso 'an Größe' u. dgl. (147 f.), sondern auch die Ausdrücke $x^{v} a r^{2} n \bar{o} x^{v} a r^{2}$ naruhastomō 'der hoheitrollste an

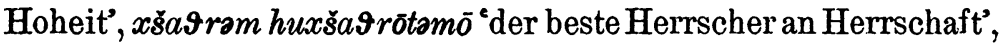
raēm raévastomō 'der reichste an Reichtum', sowie ištīm savišta 'die gewaltigsten an Besitz'.

Von den letzteren Fällen stellen sich aber wenigstens diejenigen, wo Akkusativ und Superlativ etymologisch zusammengehören, doch wohl vielmehr zum Akkusativ des Inhalts, der ja auch im Awestischen ganz geläufig gewesen sein muß (Reichelt S. $227 \mathrm{f}$.). Dieser Akkusativ ist hier ebenso adnominal

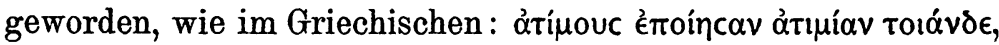

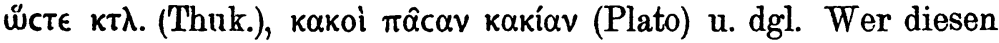
adnominalen Gebrauch freilich ebenfalls Akkusativ der Beziehung nennen will, dem läßt sich bei der Verschwommenheit dieses technischen Namens kaum widersprechen.

Weniger klar ist mir die Genesis des Akkusativgebrauchs in Yt. 5, 98 mošu pasčaēta hvōvō ištìm baon savišta "bald nachher wurden die Hrōva die gewaltigsten an Besitz'. Es scheint aber das natürlichste, auch hier auf den Akkusativ des Inhalts zurückzugehen und zwar auf diejenige Gebrauchsschattierung, in der das Verbum an sich intransitiv ist und das Substantiv im Akkusativ etwas bezeichnet, was die besondere Erscheinungsform des Vorgangs bildet, vgl. ai. rớcatē brhád bháh 'er (Agni) leuchtet hohen Glanz', aw. avó 'rvaitǐs (Beiwort der Wolken) 'in Form von Wasser strömend'. 
11.

Für die Gebrauchsarten des Akkusativs, auf die ich glaubte die Bezeichnung Akkusativ der Beziehung einschränken zu sollen, eine minder irreführende Benennung als diese zu haben, wäre recht erwünscht, weniger der Wissenschaft als der Schule wegen. Angemessener wäre, wenn er auch keineswegs das Ideal eines wissenschaftlichen Terminus darstellt, der Name Akkusativ des erklärenden Objekts, den Delbrück Grundl. der griech. Syntax 32 neben 'Akkusativ der Beziehung' anwendet. Er verführt wenigstens nicht $\mathrm{zu}$ abenteuerlicher Übersetzung. Ich selber enthalte mich eines neuen Vorschlags, weil ich der Ansicht bin, man soll einen einmal eingebürgerten wissenschaftlichen Terminus, wenn er auch noch so unzutreffend ist, nicht verdrängen wollen, wenn man einen nur besseren, aber nicht wirklich guten Ausdruck (zur Güte gehört natürlich auch Kürze) an die Stelle zu setzen weiß.

Für die Wissenschaft bleibt die Hauptsache, daß man unsern Akkusativgebrauch entwicklungsgeschichtlich richtig verstehen lernt.

Leipzig.

Karl Brugmann.

\section{Gotica.}

1. jaindwairps.

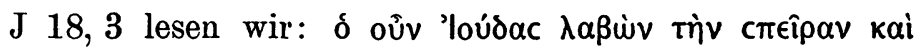

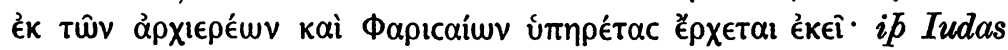
nam hansa jah pize gudjane jah Fareisaie andbahtans, iddjuh jaindwairps. Soviel ich sehn kann, fassen alle Lexikographen, auch Wrede in der 11. Auflage von Stamm-Heynes Ulfilas und Feist in dem 1909 erschienenen Wörterbuch jaindwairpss als Adverb. Der einzige Grund für diese Annahme kann nur in dem Adverb ékeî gesucht werden. Aber dieser Schluß von der griechischen Vorlage auf die gotische Übersetzung ist nicht stichhaltig. Denn mehr als einmal zeigen sich zwischen Original und Nachbildung charakteristische Unterschiede in der Wahl der Wortklasse (vgl. Stolzenburg ZZ. 37, 352 f.): Ein Substantiv vertritt den Infinitiv L 7, 21 (siuns: $\beta \lambda \epsilon ́ \pi \epsilon ı$ ) 8, 55 (mats: parєî)

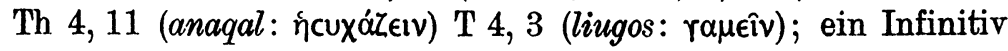

